\title{
Influence of riblet shapes on the occurrence of Kelvin-Helmholtz rollers
}

\author{
S. Endrikat ${ }^{1} \dagger$, D. Modesti $^{1}$, R. García-Mayoral ${ }^{2}$, N. Hutchins ${ }^{1}$ and \\ D. Chung ${ }^{1}$ \\ ${ }^{1}$ Department of Mechanical Engineering, University of Melbourne, Victoria 3010, Australia \\ ${ }^{2}$ Department of Engineering, University of Cambridge, Cambridge CB2 1PZ, UK
}

(Received xx; revised $\mathrm{xx}$; accepted $\mathrm{xx}$ )

We investigate turbulent flow over streamwise-aligned riblets (grooves) of various shapes and sizes. Small riblets with spacings of typically less than 20 viscous units are known to reduce skin-friction drag compared to a smooth wall, but larger riblets allow inertial-flow mechanisms to appear and cause drag reduction to break down. One of these mechanisms is a Kelvin-Helmholtz instability that García-Mayoral \& Jiménez (J. Fluid Mech., vol. 678,2011 , pp. 317-347) identified in turbulent flow over blade riblets. In order to evaluate its dependence on riblet shape and thus gain a broader understanding of the underlying physics, we generate an extensive data set comprising 21 cases using direct numerical simulations (DNSs) of fully developed minimal-span channel flow. The data set contains six riblet shapes of varying sizes between maximum drag reduction and significant drag increase. Comparing the flow fields over riblets to that over a smooth wall, we find that in this data set only large sharp-triangular and blade riblets have a drag penalty associated with the Kelvin-Helmholtz instability and that the mechanism appears to be absent for blunt-triangular and trapezoidal riblets of any size. We therefore investigate two indicators for the occurrence of Kelvin-Helmholtz rollers in turbulent flow over riblets. First, we confirm for all six riblet shapes that the groove cross-sectional area in viscous units serves as a proxy for the wall-normal permeability that is necessary for the development of Kelvin-Helmholtz rollers. Additionally, we find that the occurrence of the instability correlates with a high momentum absorption at the riblet tips. The momentum absorption can be qualitatively predicted using Stokes-flow.

Key words:

\section{Riblets and Kelvin-Helmholtz rollers}

Riblets are streamwise-aligned surface grooves with design sizes on the order of 10 viscous units that have the potential to reduce skin-friction drag compared to a smooth wall. They are classified as passive flow-control devices, as no energy input is required to favourably alter the flow field, making them attractive for aeronautical (Szodruch 1991) and maritime (Letcher et al. 1987) applications as well as for pipes (e.g. Liu et al. 1990; Peet et al. 2009). The design of riblets builds on the idea by Kramer (1937) for streamwise-aligned wires that shield a surface below from drag-increasing turbulence. Early experimental results by Liu et al. (1966) suggested the possibility of drag reduction by riblet surfaces and Walsh et. al investigated drag characteristics

$\dagger$ Email address for correspondence: s.endrikat@student.unimelb.edu.au 

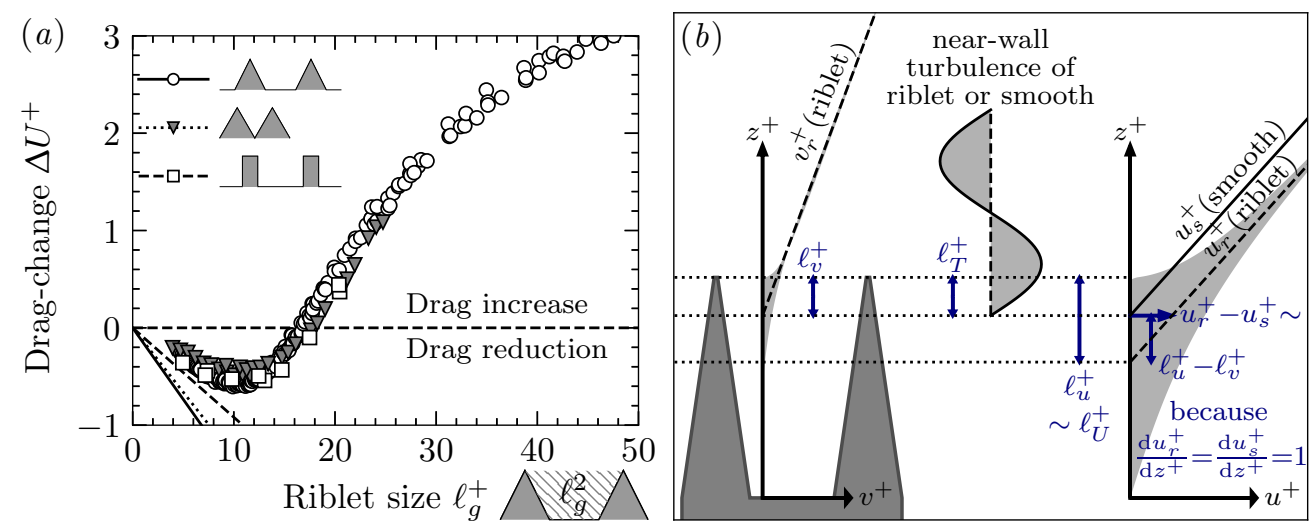

FiguRE 1. (a) Drag-change between a smooth and a riblet surface measured by a shift in the profile of mean streamwise velocity $\Delta U^{+}$for riblets of varying viscous-scaled cross-sectional area $\ell_{g}^{+2}$. The drag optimum is typically at $\ell_{g}^{+} \approx 10.7$ (García-Mayoral \& Jiménez $2011 b$ ). Data shown here are measured experimentally by Bechert et al. $(1997)\left(\nabla, R e_{\tau} \approx 250-770\right)$ and Deyn et al. (2019) ( $\left(R, R e_{\tau} \approx 150-2270\right)$ and converted from $\Delta \tau_{w} / \tau_{w, \text { smooth }}$ to $\Delta U^{+}$at matched $R e_{\text {bulk }}$. DNS data of channel flow are from García-Mayoral \& Jiménez (2012) ( $\left.\square, R e_{\tau} \approx 180-550\right)$. Straight lines indicate slopes for infinitely small riblets of the three shapes calculated in the Stokes-flow limit (Luchini et al. 1991). (b) Near-wall portion of streamwise $\left(u_{r}^{+}\right)$and spanwise $\left(v_{r}^{+}\right)$Stokes flow profiles for a riblet, where shaded regions delimit the spanwise dependence close to the surface. Protrusion heights $\ell_{u}^{+}$and $\ell_{v}^{+}$in the Stokes-flow limit approach the true solution $\ell_{T}^{+}$and $\ell_{U}^{+}$for small viscous-scaled riblet sizes.

of different riblet geometries at varying flow conditions (e.g. Walsh \& Weinstein 1978; Walsh 1982). Roughly half of the drag transport aircraft experience in cruise conditions results from skin-friction drag (e.g. Schrauf 2005), making its reduction financially and environmentally attractive. Laboratory tests of riblets have reduced skin-friction drag by up to $8.2 \%$ for friction Reynolds numbers $R e_{\tau} \equiv \delta u_{\tau} / \nu \lesssim 700$ (Bechert et al. 1997), where $\delta$ is the half-channel height, $\nu$ the kinematic viscosity and $u_{\tau} \equiv \sqrt{\tau_{w} / \rho}$ the friction velocity defined with the density $\rho$ and the wall-shear stress (drag per unit plan area) $\tau_{w}$. Following Spalart \& McLean (2011), this reduction in skin-friction drag scales to approximately $5.5 \%$ for flight conditions at $R e_{\tau} \approx 48000$ and it is only marginally affected by yawed flow up to angles of $15^{\circ}$ (Walsh \& Lindemann 1984). Possible applications explain ongoing interest by the aircraft industry and continued effort to overcome practical challenges (e.g. Bilinsky 2019; Linde \& Hegenbart 2019).

Drag reduction by riblets relative to a smooth wall at matched $R e_{\tau}$ is defined as $\mathrm{DR} \equiv 1-C_{f} / C_{f \text {,smooth. }}$. The skin friction coefficient $C_{f}=2 / U_{\delta}^{+2}$ is determined by the viscous-scaled mean streamwise velocity $U^{+}=U / u_{\tau}$ evaluated at the half-channel or boundary-layer height $\delta$. The superscript + is used to denote viscous scaling with $\nu$ and $u_{\tau}$ throughout this paper. For small differences in drag between the two surfaces, DR $\propto-\Delta U^{+}$, where $\Delta U^{+} \equiv U_{\text {smooth }}^{+}-U^{+}$is the shift between the profiles of mean streamwise velocity for the smooth and riblet surfaces at matched height in or above the logarithmic layer. Unlike a fractional drag-change, $\Delta U^{+}$is independent of the Reynolds number for large $R e_{\tau}$ and low blockage (Spalart \& McLean 2011). In figure 1(a) we observe that the drag-change $\Delta U^{+}$depends strongly on the riblet size, which we measure using the viscous-scaled square-root of the groove cross-sectional area $\ell_{g}^{+}$as proposed by García-Mayoral \& Jiménez (2011b). Small riblets repel turbulent eddies by obstructing their induced spanwise flow and thus reduce mixing of streamwise momentum and consequently skin-friction drag. The detailed description of the flow around riblets that 
explains drag-reduction mainly comes from various works of Bechert et. al and Luchini et. al and was recently reviewed by García-Mayoral et al. (2019). Luchini et al. (1991) explain the drag-reduction mechanism for riblets that are small enough for the flow around them to be dominated by viscosity, which is therefore described by Stokes flow. In this framework, the cross flow from quasi-streamwise vortices is represented by spanwise Stokes-flow $v_{r}$ (figure $1 b$ ), which penetrates the groove to a depth below the crest given by the lateral protrusion height $\ell_{v}$. Importantly, this is less than the protrusion height of streamwise Stokes-flow $\ell_{u}>\ell_{v}$. In turbulent flow, the penetration depth of Reynolds stresses given by $\ell_{T} \sim \ell_{v}$ is thus less than the streamwise protrusion height $\ell_{U}>\ell_{T}$ (Luchini 1996). If we now consider a reference smooth wall $\ell_{T}$ below the riblet crest, both flows are similar (Luchini 1996) in that their total stresses match at every height. The origins of streamwise flow of the two surfaces however differ by $\ell_{U}-\ell_{T}=\ell_{u}-\ell_{v}>0$ in the Stokes-flow limit. That difference describes the velocity decrement $\Delta U^{+} \approx \ell_{T}^{+}-\ell_{U}^{+}<0$, because both riblet and smooth-wall velocity profiles have the same slope $\mathrm{d} U^{+} / \mathrm{d} z^{+} \approx 1$ in the viscous sublayer (Luchini 1996). Consequently, $U^{+} \approx U_{\text {smooth }}^{+}-\Delta U^{+}$at every height above the reference smooth wall. In the Stokes-flow limit, riblet geometries with sharp tips (triangular and parabolic grooves) have a higher protrusion height difference than rounded sinusoidal profiles (Luchini et al. 1991). However, the protrusion height difference of finite-thickness blade riblets increases through tip rounding, because it effectively sharpens the otherwise flat tips (García-Mayoral \& Jiménez 2011a). The theoretical maximum $\ell_{u}-\ell_{v} \approx 0.13 s$, where $s$ is the riblet spacing, is attained for infinitely thin (sharp) and deep blade riblets (Luchini et al. 1991). Viscous analysis further predicts a linear decrease of drag for small riblets (straight lines in figure 1a), which implies that drag reduces with increasing riblet size until they are large enough that inertial effects contribute to momentum transport in the vicinity of the wall. For larger riblets therefore, viscous theory no longer fully captures the drag-change and we find a minimum in the drag curve (figure 1a). This breakdown of the drag-reducing regime scales with $\ell_{g}^{+}$for various riblet shapes, and the drag-minimum is typically at $\ell_{g}^{+} \approx 10.7$ (García-Mayoral \& Jiménez 2011b).

For large riblets with $\ell_{g}^{+} \gtrsim 10.7$, drag reduction degrades with increasing riblet size and for $\ell_{g}^{+} \gtrsim 17$ typical grooved surfaces have higher drag than a smooth wall (figure $1 a$ ). Even though the drag-increase has been known to occur since at least the 1980s, the physical processes responsible for the breakdown of drag reduction have thus far evaded complete understanding, which might limit our ability to design riblets that provide enough drag reduction to be economically viable for airlines. In general, drag increases as Reynolds shear stresses transport streamwise momentum into sufficiently large grooves and three main mechanisms have been proposed to explain the details of this transport. Choi et al. (1993) suggest that as the spacing of triangular riblets becomes large enough for streamwise vortices to descend into the groove, they sweep high-speed fluid towards an increased wetted area and thus enhance skin-friction drag. Goldstein \& Tuan (1998) on the other hand attribute vertical mixing to secondary mean flows created as triangular riblets deflect lateral flow into the wall-normal direction. García-Mayoral \& Jiménez (2011b) demonstrate that spanwise-aligned vortical structures related to a Kelvin-Helmholtz instability contribute to drag of blade riblets that are larger than the optimum size for drag reduction (figure $1 a$ ).

What is currently lacking is a data set comprising several riblet geometries to assess whether Kelvin-Helmholtz rollers appear also in the earlier studies. While KelvinHelmholtz rollers typically develop in free shear flows (e.g. Drazin \& Reid 2004), coherent spanwise-aligned rollers related to a Kelvin-Helmholtz instability have also been reported to appear in the flow directly above plant canopies (Raupach et al. 1996; Nepf et al. 2007). 
Furthermore, Kelvin-Helmholtz rollers contribute to turbulent mixing and thus drag of porous walls (Jiménez et al. 2001; Breugem et al. 2006; Gómez-de-Segura \& GarcíaMayoral 2019) and blade riblets (García-Mayoral \& Jiménez 2011b). The flow over natural plant canopies and artificial porous surfaces suggests two parameters that describe the formation of Kelvin-Helmholtz rollers in wall-bounded flow. First, these surfaces impede wall-normal velocity fluctuations to varying extent depending on the depth and wallnormal permeability of the substrate, which affects the ability of coherent rollers to develop in their proximity (Jiménez et al. 2001; Gómez-de-Segura \& García-Mayoral 2019). This is how García-Mayoral \& Jiménez (2011b) explain that Kelvin-Helmholtz rollers only appear over large blade riblets of sizes $\ell_{g}^{+} \gtrsim 11$. Second, a mixing layer with an inflection point in the profile of mean-streamwise velocity may be created between slow flow around the roughness elements and the faster stream above (Raupach et al. 1996). This inflection point is usually proposed as a necessary condition for instability in shear flows (Rayleigh 1879), although the linear stability analysis of smooth-wall flow with added wall-normal permeability by García-Mayoral \& Jiménez (2011b) suggests that Kelvin-Helmholtz rollers can also appear without an inflection point, because in the limit of infinite permeability, the impedance boundary condition mimics a free shear layer. Canopy drag generally increases shear in the mixing layer and thus growth of the instability, but particularly high resistance to streamwise velocity fluctuations also damps Kelvin-Helmholtz rollers (Nepf et al. 2007; Singh et al. 2016; Sharma \& GarcíaMayoral 2020b). Sparse canopies with low drag on the other hand have a weaker mixing layer and their turbulent flow field is consequently less affected by the Kelvin-Helmholtz instability (Poggi et al. 2004; Sharma \& García-Mayoral 2020a). Changing the riblet geometry therefore has the potential to not only alter the wall-normal permeability of the plane at the riblet crest, but also the mixing layer around the riblet tips from which Kelvin-Helmholtz rollers develop. For example, the blade riblets with a finite tip-width studied by García-Mayoral \& Jiménez (2011b) absorb part of the momentum at the top of the crest, where high local drag creates strong shear. This distinguishes them from other common riblet shapes with pointed tips.

In this study, we conduct direct numerical simulations (DNSs) of the flow over riblets of six different shapes, and compare them to smooth-wall flow to study how geometry differences affect the Kelvin-Helmholtz instability and thus the drag-characteristics of a riblet surface. We visualize differences in physical space (§3.1) and in spectral space (§3.2). García-Mayoral \& Jiménez (2011b) observed that the instability affects the flow in a distinct spectral region of streamwise wavelengths $65 \lesssim \lambda_{x}^{+} \lesssim 290$, which allows evaluating its influence on drag. Following the same method in $\S 4$, we quantify the dragpenalty that is due solely to the instability. In $\S 5.1$, we verify effects of wall-normal permeability on Kelvin-Helmholtz rollers for all six riblet shapes with varying viscousscaled sizes. Lastly, in $§ 5.2$, we illustrate how the shear in the mixing layer, and thus the development of the instability, depend on the riblet shape. We offer concluding remarks in $\S 6$.

\section{DNS data set of six riblet shapes and varying sizes}

We investigate the flow over six different riblet shapes with varying viscous-scaled sizes for a total of 21 cases. Four of those shapes are triangular riblets. Symmetric triangles have tip angles $\alpha=30^{\circ}, 60^{\circ}$ and $90^{\circ}\left(M \circlearrowleft \wedge\right.$, case names T3s $\left.s^{+}, \mathrm{T} 6 s^{+}, \mathrm{T}^{\circ} s^{+}\right)$making them similar to those studied by Choi et al. (1993); Bechert et al. (1997); Goldstein \& Tuan (1998) and Deyn et al. (2019). Asymmetric triangular riblets have $\alpha=63.4^{\circ}$ $\left(\Lambda, \mathrm{ATs}^{+}\right)$. Our trapezoidal riblets $\left(\Lambda \Lambda, \mathrm{TA}^{+}\right)$have the same tip angle $\alpha=30^{\circ}$ 


\section{Trapezoidal}

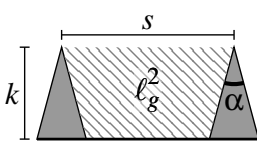

Symmetric triangular $z$

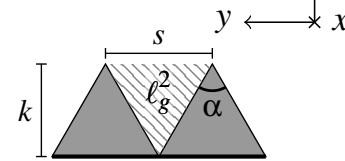

Asymmetric triangular

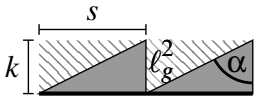

Blade

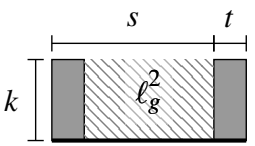

Smooth wall
Case $s^{+} \quad k^{+} \quad \ell_{g}^{+} \quad \alpha \quad \Delta x^{+}$

$\begin{array}{llllll}\text { TA18 } & 17.9 & 8.9 & 11.8 & 30.0^{\circ} & 6.0\end{array}$

$\begin{array}{llllll}\text { TA31 } & 31.3 & 15.6 & 20.6 & 30.0^{\circ} & 6.0\end{array}$

$\begin{array}{llllll}\text { TA36 } & 36.5 & 18.2 & 24.0 & 30.0^{\circ} & 6.0\end{array}$

$\begin{array}{lllllll}\text { TA50 } & 50.0 & 25.0 & 32.9 & 30.0^{\circ} & 6.0 \\ \text { TA63 } & 62.5 & 31.3 & 41.1 & 30.0^{\circ} & 6.0\end{array}$

$\begin{array}{llllll}\text { TA63 } & 62.5 & 31.3 & 41.1 & 30.0^{\circ} & 6.0\end{array}$

$\begin{array}{llllll}\mathrm{T} 310 & 10.1 & 18.8 & 9.75 & 30.0^{\circ} & 6.0\end{array}$

$\begin{array}{llllll}\mathrm{T} 321 & 21.1 & 39.4 & 20.4 & 30.0^{\circ} & 6.0\end{array}$

$\begin{array}{llllll}\mathrm{T} 333 & 33.3 & 62.2 & 32.2 & 30.0^{\circ} & 6.0\end{array}$

$\begin{array}{llllll}\mathrm{T} 615 & 14.7 & 12.7 & 9.68 & 60.0^{\circ} & 6.0\end{array}$

$\begin{array}{lllllll}\mathrm{T} 635 & 35.0 & 30.3 & 23.0 & 60.0^{\circ} & 6.0\end{array}$

$\begin{array}{llllll}\text { T919 } & 19.2 & 9.6 & 9.60 & 90.0^{\circ} & 6.0\end{array}$

$\begin{array}{llllll}\mathrm{T} 950 & 50.0 & 25.0 & 25.0 & 90.0^{\circ} & 6.0\end{array}$

$\left\{\begin{array}{cccccc}\text { AT15 } & 14.7 & 7.4 & 7.36 & 63.4^{\circ} & 6.5 \\ \text { AT19 } & 19.2 & 9.6 & 9.62 & 63.4^{\circ} & 6.5 \\ \text { AT31 } & 31.3 & 15.6 & 15.6 & 63.4^{\circ} & 6.5 \\ \text { AT42 } & 41.7 & 20.8 & 20.8 & 63.4^{\circ} & 6.5 \\ \text { AT50 } & 50.0 & 25.0 & 25.0 & 63.4^{\circ} & 6.5\end{array}\right.$$$
(\mathrm{B}
$$

$\left(\begin{array}{lllll}\text { BL20 } & 20.3 & 10.1 & 12.8 & 5.0\end{array}\right.$

$\begin{array}{lllll}\text { BL33 } & 33.3 & 16.7 & 21.1 & 5.0\end{array}$

$\begin{array}{lllll}\text { BL39 } & 39.0 & 19.5 & 24.7 & 5.0 \\ \text { BL49 } & 49.0 & 24.5 & 31.0 & 5.0\end{array}$

$6.0 \quad 0.5$

6.0

6.0

$\begin{array}{llllll}\mathrm{S} 395 & - & - & - & - & 6.0\end{array}$

S1000
$\Delta y^{+} \quad n_{s}$

$\Delta z^{+}$

$0.47-2.4 \quad 27$

$\begin{array}{lll}0.76-3.9 & 27 & 0.31-7 . \\ 0.31-7.1\end{array}$

$0.95-4.9 \quad 27 \quad 0.31-7.1$ $\begin{array}{lll}0.27-3.0 & 27 & 0.31-7.0\end{array}$

$\Delta t^{+}$

$\times 10^{3} \quad L_{x}^{+} \quad L_{y}^{+} \quad \delta^{+}$

$\begin{array}{lllll}47.4 & 2054 & 250 & 395.0 & 389.6\end{array}$

$\begin{array}{lllll}47.4 & 2054 & 250 & 395.0 & 385.5\end{array}$

$\begin{array}{lllll}47.4 & 2054 & 255 & 395.0 & 383.9\end{array}$

$\begin{array}{llllll}47.4 & 2054 & 250 & 395.0 & 379.8\end{array}$

$\begin{array}{lllll}47.4 & 2054 & 250 & 395.0 & 376.0\end{array}$

$\begin{array}{llllllll}.057-1.5 & 29 & 0.033-7.0 & 15.8 & 1027 & 252 & 395.0 & 386.3\end{array}$

$\begin{array}{cccccccc}0.12-3.2 & 29 & 0.023-6.9 & 15.8 & 1027 & 253 & 395.0 & 376.9\end{array}$

$\begin{array}{llllllll}0.83-3.4 & 41 & 0.20-8.5 & 25.0 & 2000 & 600 & 1000.0 & 971.4\end{array}$

$\begin{array}{llllllll}0.083-2.2 & 29 & 0.041-7.0 & 31.6 & 1027 & 250 & 395.0 & 390.0\end{array}$

$\begin{array}{llllllll}0.16-4.9 & 33 & 0.014-4.7 & 23.7 & 1027 & 245 & 395.0 & 383.1\end{array}$

$\begin{array}{llllllll}0.11-2.9 & 29 & 0.047-7.1 & 47.4 & 1027 & 250 & 395.0 & 392.2\end{array}$

$\begin{array}{lllllllll}0.30-7.1 & 33 & 0.029-7.0 & 47.4 & 1027 & 250 & 395.0 & 387.8\end{array}$

$\begin{array}{llllllll}0.23-2.0 & 26 & 0.40-5.8 & 47.4 & 1027 & 250 & 395.0 & 392.6\end{array}$

$\begin{array}{llllllll}0.37-1.9 & 28 & 0.40-5.8 & 47.4 & 1027 & 250 & 395.0 & 391.9\end{array}$

$\begin{array}{llllllll}0.15-4.3 & 55 & 0.40-5.7 & 47.4 & 1027 & 250 & 395.0 & 390.0\end{array}$

$\begin{array}{llllllll}0.45-2.7 & 46 & 0.40-4.9 & 47.4 & 1027 & 250 & 395.0 & 388.3\end{array}$

$\begin{array}{llllllll}0.32-1.7 & 63 & 0.40-4.4 & 47.4 & 1027 & 250 & 395.0 & 387.0\end{array}$
$L_{t} u_{\tau} / \delta$

50.1

46.7

49.2

45.7

46.7

69.3

65.0

19.1

91.1

93.5

127

95.0

45.4

121

47.5

117

60.3

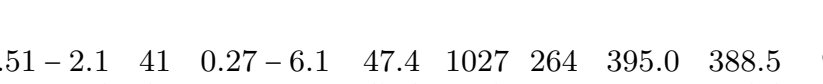

$\begin{array}{lllllllll}83-3.4 & 41 & 0.28-6.3 & 47.4 & 1027 & 266 & 395.0 & 384.4 & 94.6\end{array}$

$1.2-4.9$

3.0

3.0 $\begin{array}{lllllll}1 & 0.30-6.7 & 47.4 & 1027 & 294 & 395.0 & 379.3\end{array}$

93.3

118

$\begin{array}{llllllll}- & 0.21-4.7 & 79.0 & 1027 & 250 & 395.0 & - & 173 \\ - & 0.32-8.8 & 80.0 & 2000 & 600 & 1000.0 & - & 58.5\end{array}$
$\Delta U^{+} \pm \zeta^{+} \quad \varepsilon^{\prime+}$

$-1.06 \pm 0.10 \quad 4.2$

$0.44 \pm 0.10 \quad 3.4$

$0.81 \pm 0.10 \quad 4.5$

$1.76 \pm 0.10 \quad 5.9$

$2.47 \pm 0.10 \quad 7.3$

$-0.77 \pm 0.12 \quad 6.1$

$0.83 \pm 0.12 \quad 5.4 \stackrel{ }{2}$

$2.75 \pm 0.10 \quad 8.4$ ङे.

$-0.82 \pm 0.10 \quad 3.3$ $0.64 \pm 0.10 \quad 4.1$

$-0.61 \pm 0.08 \quad 4.7$ $0.78 \pm 0.10 \quad 7.8$

ㅇำ

$-0.50 \pm 0.143 .1$ స

$0.50 \pm 0.09 \quad 3.4$ ริ

$0.22 \pm 0.09 \quad 2.4$

$0.49 \pm 0.12 \quad 1.6$

$-0.60 \pm 0.10 \quad 3.0$

$0.58 \pm 0.10 \quad 3.0$

$1.19 \pm 0.10 \quad 3.1$

$1.84 \pm 0.09 \quad 2.9$

- 3.6

$-\quad 3.6$
$-\quad 3.3$

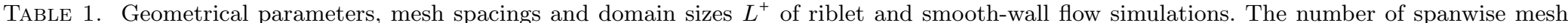

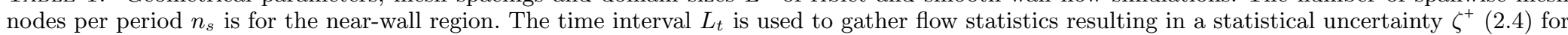

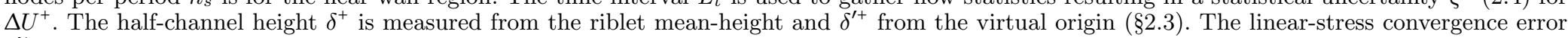
$\varepsilon^{\prime+}$ is defined in (2.5). 

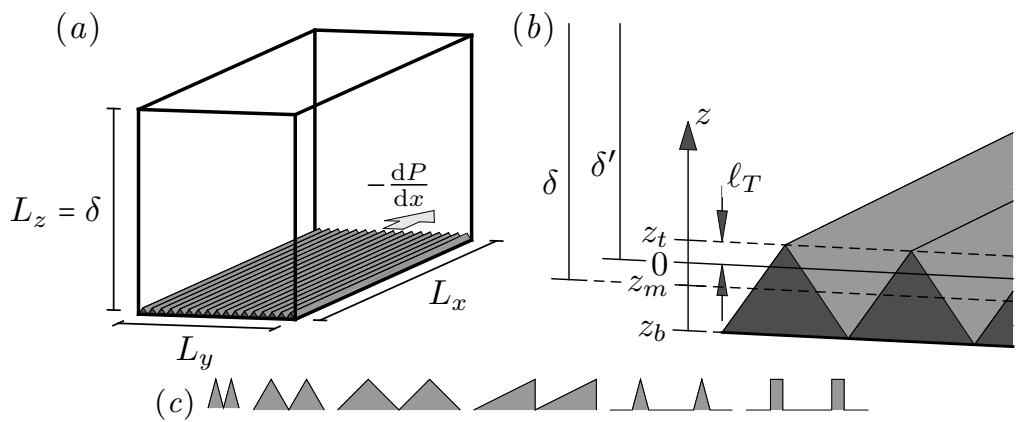

Figure 2. Minimal-span $\left(L_{y} \ll \delta\right)$ open-channel computational domain with triangular riblets. Full view in $(a)$ and close-up of the surface in $(b)$, with $\delta$ measured from the mean-height $z_{m}$ and $\delta^{\prime}$ from the virtual origin for turbulence at $z=0(\S 2.3)$. Riblet tips are at $z_{t}$ and the groove bottom is at $z_{b}$. (c) Sketches of the six riblet shapes in the spanwise wall-normal plane.

that Bechert et al. (1997) used, and are therefore a bit sharper than those from Deyn et al. (2019) with $\alpha=53.5^{\circ}$. We further consider blade riblets with a spacing-to-thickness ratio $s / t=5$ (几几, BL $s^{+}$) that are similar to the blades by García-Mayoral \& Jiménez $(2011 b, 2012)$ with $s / t=4$. The groove depth $k$ is half of its spacing $s$, except for the triangular riblets with $\alpha=30^{\circ}$ and $60^{\circ}$. Simulation parameters are summarised in table 1 .

\subsection{Numerical Setup}

We conduct DNSs to solve the Navier-Stokes equations for an incompressible fluid

$$
\frac{\partial \mathbf{u}}{\partial t}+\nabla \cdot(\mathbf{u u})=-\frac{1}{\rho} \nabla p+\nu \nabla^{2} \mathbf{u}-\frac{1}{\rho} \frac{\mathrm{d} P}{\mathrm{~d} x} \mathbf{e}_{\mathbf{x}}, \quad \nabla \cdot \mathbf{u}=0,
$$

in open (one-sided) channels that have a no-slip smooth (flat) wall or riblet surface on the bottom and a free-slip wall with symmetry boundary conditions at the top (figure 2). Based on the similarity of near-wall flows across internal and external flows (e.g. Monty et al. 2009), we can expect the riblet flow in one-sided channels to be representative of that in boundary layers, where riblets are commonly applied. Periodic boundary conditions are applied in both wall-parallel directions. The half-channel height $\delta$ is measured from the riblet mean-height to the top of the open channel, such that the cross-sectional area matches that of the smooth-wall channel. The velocity $\mathbf{u}$ has components $u, v$ and $w$ in the streamwise $(x)$, spanwise $(y)$ and wall-normal $(z)$ directions respectively and $t$ represents time. Fluctuations are defined as deviations from the temporal, streamwise and riblet-period mean $u^{\prime}(x, y, z, t)=u(x, y, z, t)-U(z)-\widetilde{u}(y, z)$, where $U=\bar{u}$ is the $x$ $y$-t-average at each height $z$ and $\widetilde{u}$ are dispersive or form-induced fluctuations $(\overline{\widetilde{u}}=0)$ that are invariant with the streamwise direction for 2D riblets and found by averaging over $x, t$ and riblet periods. Spanwise averages below the riblet tips are superficial, i.e. they include solid regions with zero velocity, to avoid a jump in intrinsic averages at the crest, particularly for blade riblets. Pressure is decomposed into the $x-y$-periodic component $p$ and the driving contribution $P$, whose gradient acts along the unit vector in the streamwise direction $\mathbf{e}_{\mathbf{x}}$. Therefore, the time-averaged wall-shear stress integrates to $\tau_{w} / \rho=-(\delta / \rho) \mathrm{d} P / \mathrm{d} x$, where the parameters $\tau_{w} / \rho, \delta$ and $(1 / \rho) \mathrm{d} P / \mathrm{d} x$ are held constant across all cases. The friction Reynolds number $R e_{\tau}=\delta u_{\tau} / \nu=395$ for all but one riblet case with $R e_{\tau}=1000$ (table 1 ).

We solve (2.1) using the incompressible second-order accurate finite-volume flow solver Cliff by Cascade Technologies Inc. (Ham et al. 2006, 2007). Variables are collocated at the nodes of unstructured meshes and time marching is based on the fractional-step method 
(e.g. Kim \& Choi 2000) with a constant step size $\Delta t$ (table 1) chosen small enough to ensure that the maximum convective CFL number is generally below 1 . Time-averages of the maximum CFL numbers for the present cases are in the range $0.38-0.76$ and fluctuations above 1 occurred for about $1.4 \%$ of the time steps.

Near-wall portions of meshes for the four riblet types are shown in figure 3 . The wallnormal mesh with $n_{z}$ nodes is non-uniform according to

$$
z_{i}=\frac{1}{2} \tanh \left(\xi_{i} \tanh ^{-1}(\alpha)\right) \quad \text { with } \quad \xi_{i}=\frac{2(i-1)}{n_{z}-1}-1, \quad \text { where } i=1,2, \ldots, n_{z}
$$

as proposed by Moin \& Kim (1982) with $\alpha=0.978$ for simulations at $R e_{\tau}=395$ and $\alpha=0.982$ at $R e_{\tau}=1000$. For triangular riblets (figure $3 a$ ), a smooth-wall mesh with this spacing is conformally mapped to the geometry. For trapezoidal (figure $3 b$ ) and blade (figure $3 c$ ) riblets, the node distribution according to $(2.2)$ starts at the tip height $z_{t}$. The mesh in the groove is mirroring that wall-normal spacing to ensure a high resolution around the riblet tips. At least 26 nodes per riblet period are used in the spanwise direction to resolve the geometry (table 1), which is finer than the spanwise mesh necessary to capture relevant turbulent flow structures over a smooth wall. The asymmetric triangular riblets in figure $3(d)$ are meshed using the algorithm Adapt by Cascade Technologies Inc., for which maximum spacings in every direction are prescribed for individual regions resulting in the spacings given in table 1 . In the case of a smooth wall, the streamwise mesh is sufficiently fine, as a refinement from our present $\Delta_{x}^{+}=6$ to $\Delta_{x}^{+}=4$ (not shown) does not change spectra of Reynolds shear stress, wall-normal and streamwise velocity. As a reference, García-Mayoral \& Jiménez (2012) use $\Delta_{x}^{+} \approx 6$ at $R e_{\tau} \approx 180$ and $\Delta_{x}^{+} \approx 9$ at $R e_{\tau} \approx 550$ for blade riblets with a spectral solver. A mesh refinement study (Endrikat et al. 2020) in all three directions for triangular riblets confirms that velocity fluctuations are resolved across all relevant scales and that $\Delta U^{+}$ is approximately mesh independent. For example, we made the mesh finer by factors of about $1.5,1.3$ and 1.5 in $x, y$ and $z$ relative to our present mesh in figure $3(a)$ and observed an increase in $\Delta U^{+}$of 0.02 , which cannot be discerned from the statistical uncertainty $\Delta U^{+} \pm 0.1$ that we set to control the runtime of our simulations (details below). We also coarsened the mesh by factors of about $2,1.6$ and 2 in $x, y$ and $z$ relative to that in figure $3(a)$ and found that fluctuations of Reynolds stresses, considered separately at different wavelengths, only deviate from the solution on the finer present mesh for heights $z^{+}-z_{t}^{+} \gtrsim 20$. The mean flow and fluctuations in and just above the groove might therefore be over-resolved on the meshes from our present study, which gives us confidence that they accurately describe the surface geometry and the flow around it.

All simulations for this study employ the minimal-span channel concept. First conceived as a numerical experiment for understanding the structure of near-wall turbulence over a smooth wall (Jiménez \& Moin 1991; Flores \& Jiménez 2010; Hwang 2013), it revealed that flow close to the wall is only marginally affected by unphysically narrow computational domains. For rough-wall channels, Chung et al. (2015) and MacDonald et al. (2017) demonstrated that the minimal-span channel can be used to accurately determine the drag-change of a given surface with respect to a smooth wall, while significantly reducing the computational cost compared to traditional full-span channel simulations. In minimal-span channels, the velocity profile diverges from that of a fullspan channel for heights $z^{+}>z_{c}^{+}$, which Flores \& Jiménez (2010) find depends on the spanwise domain extent $z_{c}^{+} \approx 0.3 L_{y}^{+}$. Later (Hwang 2013; Chung et al. 2015) determine less conservatively $z_{c}^{+} \approx 0.4 L_{y}^{+}$, provided that this location is in the logarithmic layer. Consequently, the shift in the profile of mean streamwise velocity $\Delta U^{+}$, used to measure a drag-change, needs to be evaluated at $z^{+} \leq z_{c}^{+}$in the log-layer to obtain a result that 

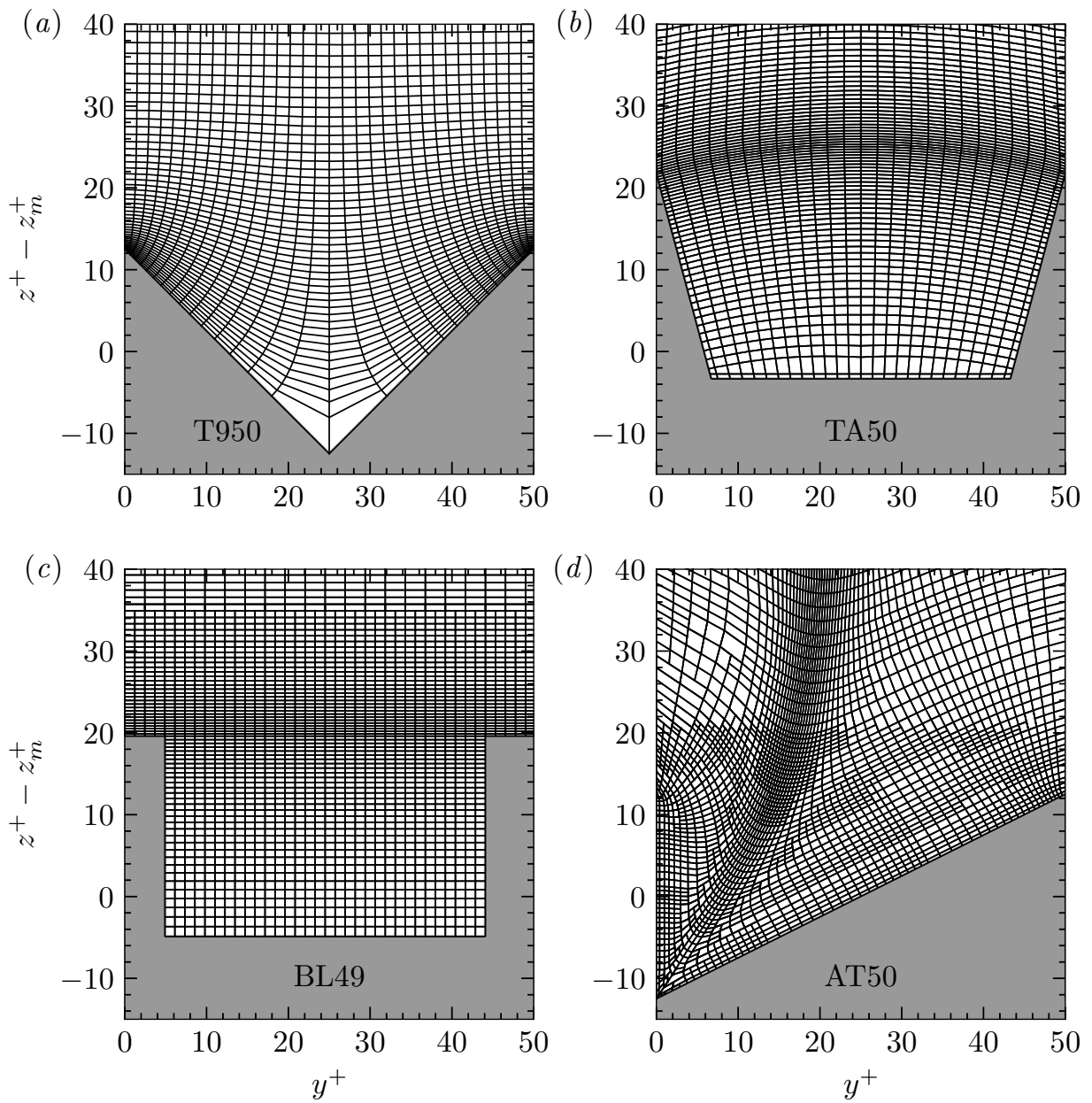

FiguRE 3. Near-wall cross-sections of representative computational meshes for one period of the triangular $(a)$, trapezoidal $(b)$, blade $(c)$ and asymmetric triangular $(d)$ riblets. The number of points per riblet span $n_{s}$ in table 1 is for the near-wall region after refinement. Meshes are coarsened towards the top of the domain in both spanwise and wall-normal directions $\left(\Delta y^{+}\right.$and $\Delta z^{+}$in table 1), except for the symmetric triangular riblets $(a)$ that only stretch in $z$. Meshes for the trapezoidal riblets $(b)$ are coarsened in $y$ at $z-z_{t}=2.5 k$, where they are spanwise uniform.

does not depend on the channel width. Furthermore, we need to measure $\Delta U^{+}$above the roughness sublayer in order to capture all effects of the surface. Consistent with sinusoidal roughness (Chan et al. 2018), we can expect the roughness sublayer to be limited to $z^{+} \lesssim z_{\mathrm{RSL}}^{+} \approx 0.5 s^{+}$, where $s^{+}$is the riblet spacing. Following MacDonald et al. (2017), the spatial domains of our channels are constrained by

$$
L_{x}^{+} \gtrsim \max \left(3 L_{y}^{+}, 1000\right), \quad z_{c}^{+}=0.4 L_{y}^{+} \gtrsim 0.5 s^{+} .
$$

We chose $L_{y}^{+} \approx 250$ at $R e_{\tau}=395$ such that $z_{c}^{+} \approx 100$ and $L_{y}^{+}=600$ at $R e_{\tau}=1000$ such that $z_{c}^{+}=240$, which is inside the log-layer and above the height of the roughness sublayer. For the present code we varied the channel width $L_{y}^{+}=\{150,250,450\}$ at $R e_{\tau}=395$ (Endrikat et al. 2020), to verify that our minimal channels accurately resolve both the mean flow and fluctuations of size $\lambda_{x}^{+}<L_{x}^{+}$and $\lambda_{y}^{+}<L_{y}^{+}$for $z^{+}-z_{t}^{+} \lesssim 30$, in agreement 
with MacDonald et al. (2017). The same conclusions hold when we compare the flow over blade riblets in minimal-span channels to those from full-span channels by GarcíaMayoral \& Jiménez (2012). Our domains with $L_{y}^{+} \approx 250$ resolve all relevant fluctuations in the spectral and wall-normal region that may be affected by the Kelvin-Helmholtz instability and the energy in that spectral region matches that in wider domains, both for flows with and without Kelvin-Helmholtz rollers (Endrikat et al. 2020). Therefore, the constraint of the largest scales in the flow does not seem to affect the occurrence or strength of Kelvin-Helmholtz rollers, whose energy accumulates partly in the spanwise infinite wavelength of minimal-span channels.

For flows with $z_{c}^{+}$in the log-layer, the minimum simulation time $L_{t}$ following initial transients that is required to reach a $95 \%$ confidence interval for the roughness function $\Delta U^{+} \pm \zeta^{+}$can be estimated according to MacDonald et al. (2017),

$$
L_{t} \frac{u_{\tau}}{\delta} \approx\left(\frac{91.4}{\zeta^{+} z_{c}^{+}}\right)^{2} \frac{7.5 z_{c}}{L_{x}} \frac{2.5 z_{c}}{L_{y}} \frac{6 z_{c}}{L_{z}} .
$$

The uncertainty $\zeta^{+}$is given in table 1 . In channel flow, the time-averaged total stress profile is linear and we evaluate convergence of statistics using the departure from the ideal profile as suggested by Vinuesa et al. (2016),

$$
\varepsilon^{\prime+}=\left(\frac{1}{\delta^{+}-z_{t}^{+}} \int_{z_{t}^{+}}^{\delta^{\prime+}} \varepsilon^{+2} \mathrm{~d} z^{+}\right)^{1 / 2}, \quad \text { where } \varepsilon^{+}=\frac{\delta^{\prime+}-z^{+}}{\delta^{+}}+{\overline{u^{\prime} w^{\prime}}}^{+}+\overline{\widetilde{u}}^{+}-\frac{\mathrm{d} U^{+}}{\mathrm{d} z^{+}} .
$$

For riblet channels, the stress balance defining $\varepsilon^{+}$is only valid above the crest $z_{t}$ (figure $2 b$ ), which defines the lower integration bound for $\varepsilon^{\prime+}$. Vinuesa et al. (2016) report typical values for $\varepsilon^{\prime+}$ in smooth-wall full-channel flow at $R e_{\tau} \approx 395$ by Moser et al. (1999); Iwamoto et al. (2002) as $\varepsilon^{\prime+}=4.8 \times 10^{-3}$ and $\varepsilon^{\prime+}=9.4 \times 10^{-4}$ respectively. Convergence values $\varepsilon^{\prime+}$ for the present cases in table 1 are similar to those of the referenced studies.

\subsection{Profiles of mean velocity and turbulence intensities}

Profiles of mean streamwise velocity, Reynolds shear stress and streamwise velocity variance are shown in figure 4 for all riblet cases and the reference smooth wall (......). All cases of each riblet shape are shown in the same panel, where dashed lines are used for drag-reducing riblets and solid lines for drag-increasing cases. Comparing streamwise velocity in the left column of figure 4 to the smooth wall reference, drag-reducing cases have a higher velocity $\left(\Delta U^{+}=U_{\text {smooth }}^{+}-U^{+}<0\right)$ and drag-increasing cases a lower velocity $\left(\Delta U^{+}>0\right)$ at the height $z_{c}^{+}$, above which flow in minimal-span channels is unphysical.

Velocity fluctuations in minimal-span channels depart from those in full-span channels starting at a lower height than the mean velocity that likewise depends on $L_{y}^{+}$(MacDonald et al. 2017). The near-wall portion however, that is representative of full-span channel flows, shows a peak in average Reynolds shear stress $-{\overline{u^{\prime} w^{\prime}}}^{+}$(figure 4, centre column) and streamwise velocity fluctuations $u_{\mathrm{rms}}^{+}$(figure 4 , right column). In figure 4 , the origin of the wall-normal coordinate is at the riblet mean-height and the peak of turbulence quantities is shifted upwards as the riblet size increases. For small riblets, this displacement of turbulent structures reduces momentum transfer towards the wall and consequently drag, as envisioned by Luchini (1996). For larger riblets however, drag increases nevertheless (positive $\Delta U^{+}$as shown in the left column of figure 4 ).

\subsection{Virtual origin}

We need to account for the virtual origin of the velocity profile so that its decrement $\Delta U^{+}$becomes a measure of the drag-change that is independent of the Reynolds number 

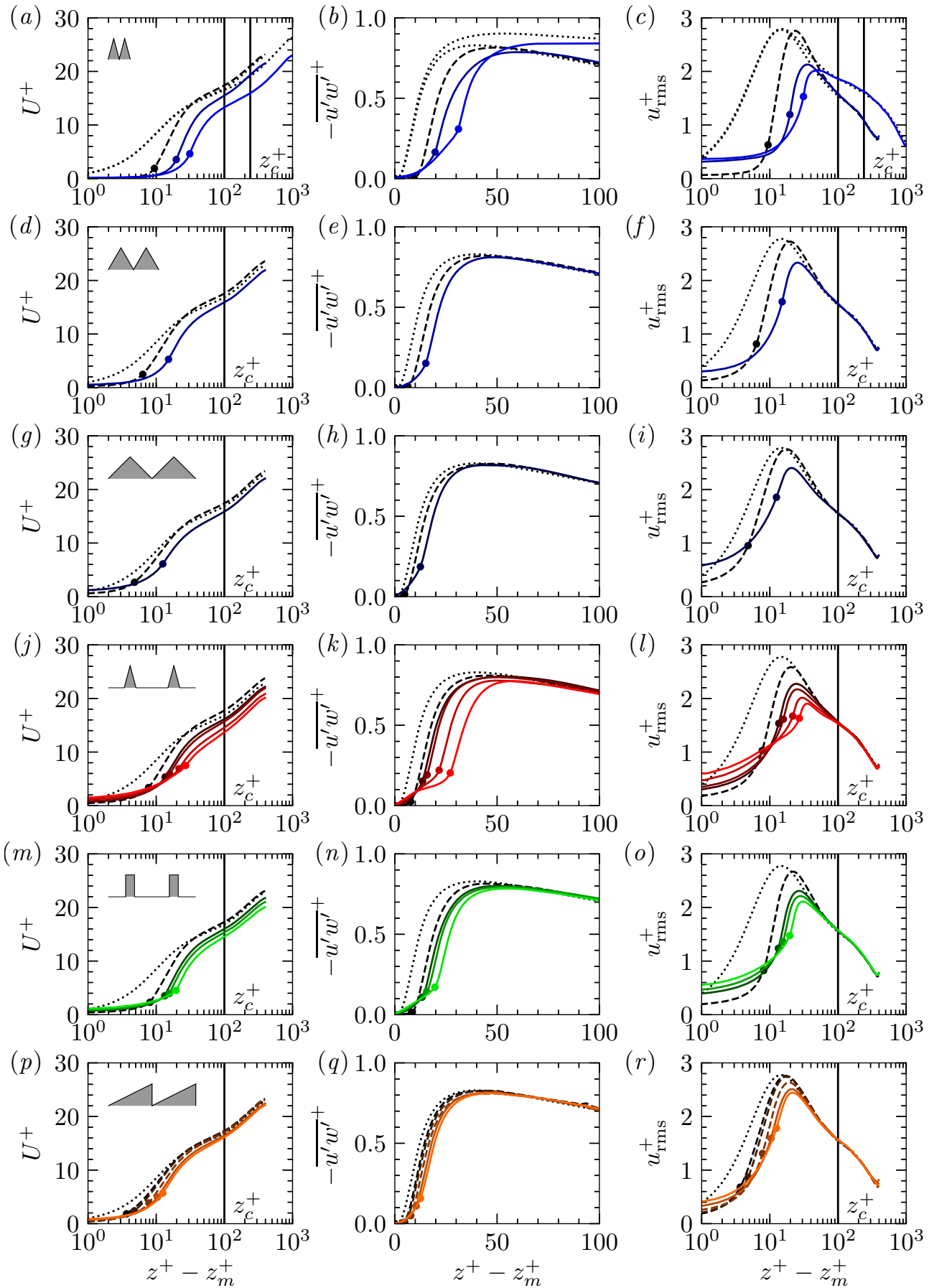

FigURE 4. Superficially averaged streamwise velocity profiles $U^{+}$, Reynolds shear stresses $-\overline{u^{\prime} w^{\prime}}$ and streamwise velocity fluctuations $u_{\mathrm{rms}}^{+}=\left({\overline{u^{\prime 2}}}^{+}\right)^{1 / 2}$. Profiles originate at the mean-height $z_{m}^{+}$ and line colours get lighter with increasing viscous-scaled riblet size and corresponding higher crest $(\bullet)$. The height $z_{c}^{+}$up to which data are representative of full-span channel flow is marked by vertical lines. Smooth wall reference data $(\cdots \cdots)$, riblets that reduce $(----)$ or increase $(-)$ drag. The friction Reynolds number $R e_{\tau}=395$, except for the largest sharp triangular riblet case in $(a-c)$ with $R e_{\tau}=1000$. 

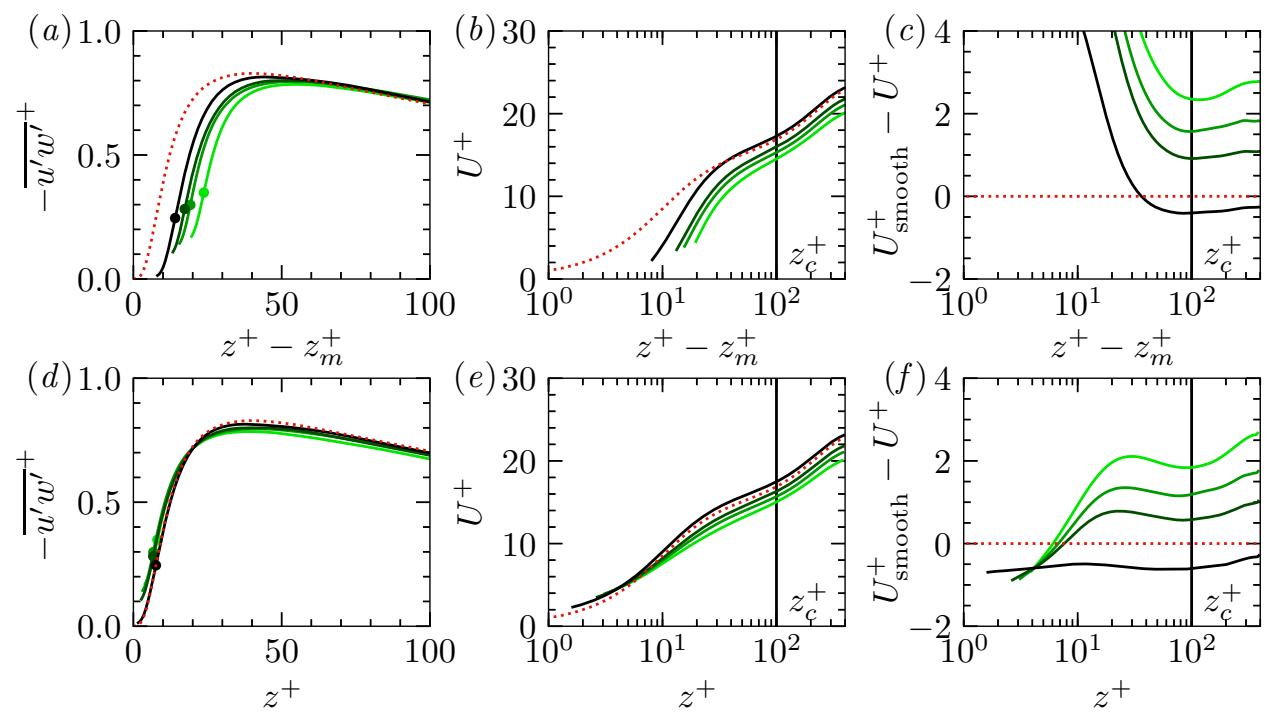

Figure 5. Profiles for blade riblet cases with $s^{+}=[20,33,39,49]$ (dark to light) and a smooth wall $(\cdots \cdots$.$) with the origin at the mean-height (a-c)$ and at the origin of turbulence defined by $\overline{u^{\prime} w^{\prime}}{ }^{+}$of the smallest riblets $(d-f)$. Profiles start at the riblet crest and are representative of full-span channel flows below $z_{c}^{+}=100$. Dots $(\bullet)$ in $(a)$ and $(d)$ mark the point of largest slope for the riblet flow profiles.

(García-Mayoral et al. 2019). This allows us to then draw conclusions regarding dragincreasing mechanisms that generalise to higher $R_{\tau}$ (Spalart \& McLean 2011). Accounting for the correct origin is particularly important at our low Reynolds number $R e_{\tau}=395$, because we measure $\Delta U^{+}$in the log-layer (at $z_{c}^{+}=100$ in minimal-span channels, §2.1), where the velocity profiles have a slope $\mathrm{d} U^{+} / \mathrm{d} z^{+} \approx 0.025$. Therefore, shifting two profiles by for example 2 viscous units with respect to each other changes the measured $\Delta U^{+}$ by about 0.05 . At higher Reynolds numbers, $\Delta U^{+}$is measured farther from the wall in viscous units, where the slopes of the velocity profiles are smaller such that the choice of origin has a lesser effect on $\Delta U^{+}$.

In order to define the origin, we assume that drag-reducing riblets can be considered rough walls with surface features that are small relative to near-wall flow structures. The outer layers of such flows are similar and the only effect of roughness is captured by $\Delta U^{+}$(Clauser 1956). Based on that assumption, Luchini (1996) suggests we consider a reference smooth wall for riblets at the origin perceived by near-wall turbulent eddies. We determine the location of the perceived smooth wall by finding the value of Reynolds shear stress in the point of largest slope of the profile (• in figure $5 a$ ) and asking at what height smooth-wall flow attains that same value. Over large riblets, turbulent eddies might not perceive the equivalent origin as a homogeneous boundary, and hence the concept of the virtual origin based on the profile of Reynolds shear stress might not be applicable in these cases (García-Mayoral et al. 2019). For a consistent definition of the origin across riblet sizes, we therefore determine the distance between the origin and the riblet crest $\ell_{T}^{+}$for riblets near the drag-optimum and hold $\ell_{T} / k$ constant across all sizes for a given riblet shape. Profiles of turbulent Reynolds shear stress after adjusting the origin are shown in figure $5(d)$. The profile for the smallest riblets closely matches that for the smooth wall as recognised by Luchini (1996) and in accordance with full similarity. Only minor differences between the two curves are noticeable in the range 
$40 \lesssim z^{+} \lesssim 60$, because these riblets with $\ell_{g}^{+}=12.8$ are not strictly in the viscous regime. For the larger riblets, differences compared to the smooth-wall profile above the peak of $-{\overline{u^{\prime} w^{\prime}}}^{+}$in figure $5(d)$ are due to a mismatch of effective Reynolds numbers after adjusting the origin, $\delta^{\prime+}<\delta^{+}$(table 1 ). Closer to the wall, in the roughness sublayer, deviations for the larger riblets suggest that flows are not similar to the smooth-wall reference at the virtual origin, because not all effects of large riblets on the flow can be captured by an origin shift alone (García-Mayoral et al. 2019). With the approach to keep $\ell_{T} / k$ constant for each shape, outer-layer similarity in terms of the mean velocity profiles (above the roughness sublayer) can be observed for all riblet sizes as seen in figure $5(f)$, where velocity profiles become parallel to that of the smooth wall by accounting for the virtual origin. At $z^{+}=z_{c}^{+}$, where the shift $\Delta U^{+}$for a given geometry is measured, figure $5(f)$ demonstrates that the slope of $U_{\text {smooth }}^{+}-U^{+}$is small and thus provides an accurate measure of the drag-change, particularly for the small riblets.

\section{Kelvin-Helmholtz instability at the riblet crest}

\subsection{Visualisation in physical space}

In order to assess the presence of spanwise elongated structures compatible with a Kelvin-Helmholtz instability, we first qualitatively analyse the instantaneous flow field. We can expect a spanwise extent of Kelvin-Helmholtz rollers of up to 1000-1500 viscous units in flow over blade riblets (García-Mayoral \& Jiménez 2012). Therefore, they are likely wider than the present minimal-span channels with $L_{y}^{+} \approx 250$. However, consistent with conclusions by (García-Mayoral \& Jiménez 2012), we show in $\S 3.2$ that the spanwise constraint of the domain does not affect the resolved wavelengths of the instability by comparing to full-span channel DNS. Although Kelvin-Helmholtz rollers develop at the riblet crest, their fluctuations extend into the groove as shown by Sharma \& García-Mayoral (2020b) for filament canopies. Therefore, footprints of Kelvin-Helmholtz rollers are readily visible in the local wall-shear stress on the surface of large triangular riblets T321 with $\alpha=30^{\circ}$ in figure $6(c)$. Negative wall-shear stress in adjacent grooves suggests the presence of spanwise coherent structures in the overlying flow that have an average streamwise spacing of about 200 viscous units. The large drag-increasing triangular $\left(\alpha=60^{\circ}\right)$, trapezoidal and blade riblets in figure $6(e, i, k)$ show similar but weaker footprints, while the large blunt triangular riblets $\left(\alpha \approx 63^{\circ}\right.$ and $\left.\alpha=90^{\circ}\right)$ in figure $6(g, m)$ have few and unconnected patches of negative wall-shear stress. In early DNS of riblet flow, Chu \& Karniadakis (1993) report local flow reversal in a plane below the crest of small triangular riblets with $\alpha \approx 53^{\circ}$ and $\ell_{g}^{+} \approx 12.1$ at $R e_{\tau} \approx 86$. Similarly, our small drag-reducing triangular $\left(\alpha=30^{\circ}, \alpha=60^{\circ}\right)$ and blade riblets in figure $6(b, d, j)$ experience local reverse flow in some grooves. These patches of negative wall-shear stress have a much less pronounced spanwise coherence than on the larger and sharp triangular riblets in figure $6(c)$, but they are nevertheless much wider than their streamwise extent and are presumably the result of weak Kelvin-Helmholtz rollers. This agrees with the observation by García-Mayoral \& Jiménez (2011b) that for small riblets with $\ell_{g}^{+} \lesssim 11$, the instability is weak and has a negligible effect on drag. The smooth wall (figure $6 a$ ) and the remaining small riblets in figure $6(f, h, l)$ do not experience significant reverse flow.

Wall-shear stress on the large and sharp triangular riblets T321 with $\alpha=30^{\circ}$ (figure $6 c$ ) shows the most prominent spanwise coherence, which suggests the presence of KelvinHelmholtz rollers in the overlying flow. Therefore, we visualise fluctuations of pressure for that case in figure 7 . The standard deviation of pressure is unphysically amplified in minimal-span channels, but the structures shown here are shorter than $3 L_{y}^{+}$in the 


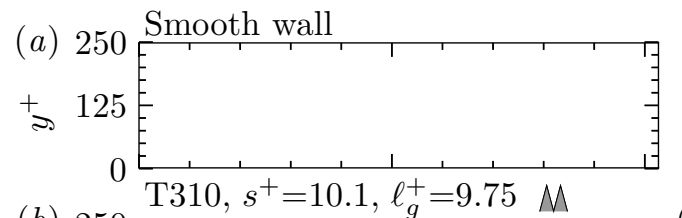

Local instantaneous wall-shear stress $\tau_{w, l} / \tau_{w}$
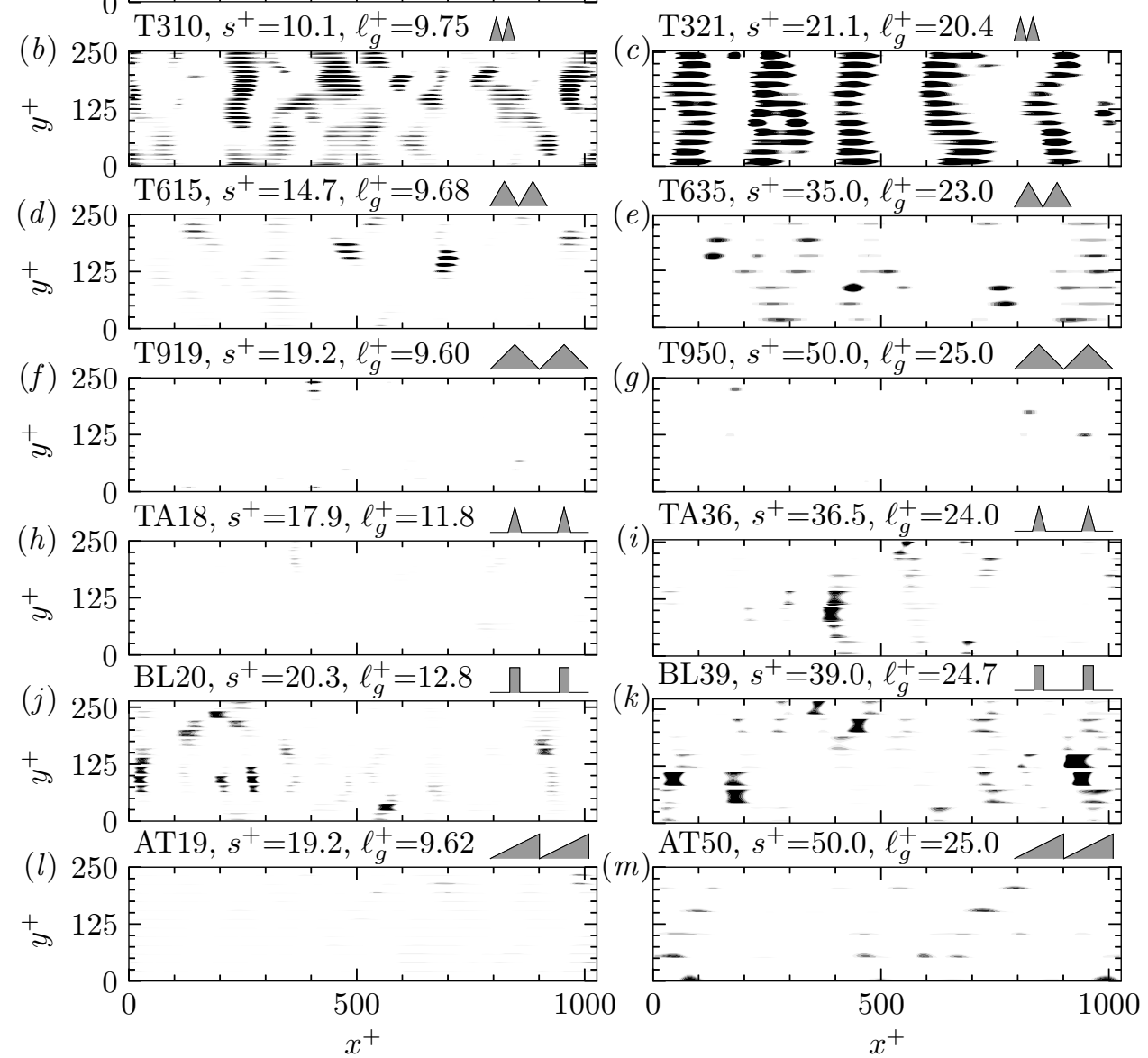

Figure 6. Local instantaneous wall-shear stress on wall segments (computational faces) with wetted area $A_{w}(x, y)$ and plan area $A_{p}(x, y), \tau_{w, l}(x, y)=\nu \rho(\partial u / \partial n) A_{w} / A_{p}$, where $n$ is the locally wall-perpendicular direction, relative to the average wall-shear stress on the entire wetted area $A, \tau_{w}=\int_{L_{t}} \int_{A} \nu \rho(\partial u / \partial n) \mathrm{d} A \mathrm{~d} t /\left(L_{x} L_{y} L_{t}\right)$. On a smooth wall $(a)$ and along various riblet surfaces $(b-m)$ to visualise reverse flow close to the surface.

streamwise direction and therefore not affected (MacDonald et al. 2017). In the spanwise average of pressure fluctuations just above the riblet tips in figure $7(a)$, small scales are averaged out and only spanwise extended eddies are visible. Many of these low-pressure regions at this instance are confined to roughly the first 25 viscous units above the crest, in agreement with the location of Kelvin-Helmholtz rollers found by García-Mayoral \& Jiménez $(2011 b)$.

We further focus on the temporal evolution of spanwise averaged pressure fluctuations in a plane 3 viscous units above the riblet crest (figure $7 b$ ) and a smooth wall (figure $7 d$ ). In the flow over a smooth wall, large structures travelling downstream with a convection velocity of about $16 \mathrm{u}_{\tau}$ (parallel to …..) leave a low-pressure mark in this plane close to the surface, but they likely originate far $\left(\gtrsim 50 \nu / u_{\tau}\right)$ from the wall (figure $7 c$ ). Low-pressure 

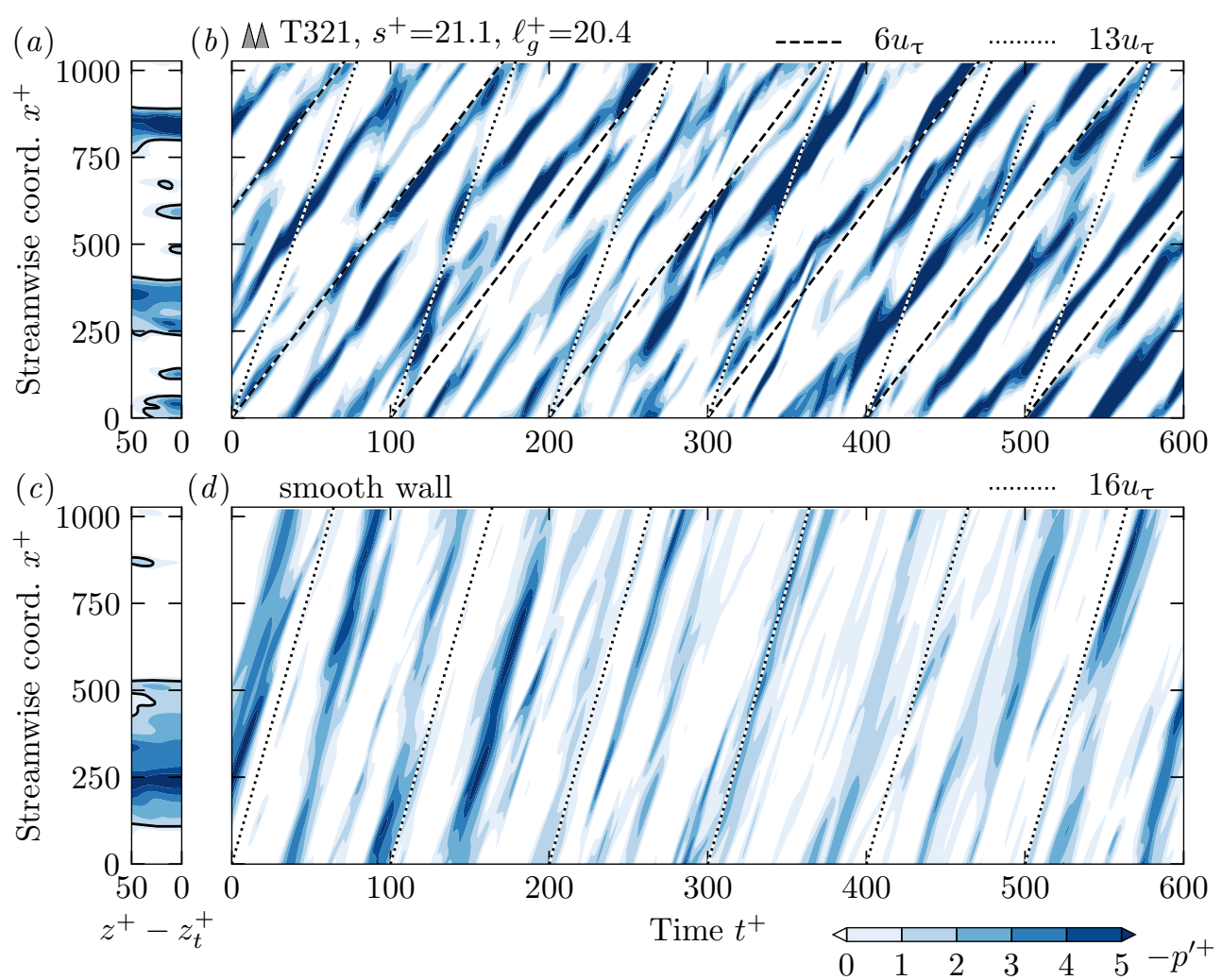

FiguRE 7. Instantaneous spanwise-averaged fluctuations of pressure. Shown against distance from the crest of large triangular riblets T321 with $\alpha=30^{\circ}(a)$ and a smooth wall $(c)$. Time evolution at one height 3 viscous units above the crest $(b)$ and smooth wall $(d)$. Flow fields are sampled every $0.79 \nu / \mathrm{u}_{\tau}^{2}$ after the flow has become statistically stationary. Time in $(b, d)$, starts at the reference time used in $(a, c)$. Lines indicate constant convection velocities: in $(b)$ $6 \mathrm{u}_{\tau}(----)$ and $13 \mathrm{u}_{\tau}(\cdots \cdots)$, in $(d) 16 \mathrm{u}_{\tau}(\cdots \cdots)$.

regions above the sharp riblets T321 in figure $7(b)$ are dominated by structures with a much lower and fairly constant velocity of about $6 \mathrm{u}_{\tau}$ (parallel to ----). This convection velocity is similar to the $6 \mathrm{u}_{\tau}$ to $8 \mathrm{u}_{\tau}$ of Kelvin-Helmholtz rollers over blade riblets (García-Mayoral \& Jiménez 2011b) and it is consistent with an expected convection velocity between that of the low-speed side of the mixing layer (in the groove) and the high-speed side (some 20-30 viscous units above). The average flow speed at the riblet tips $U_{t}^{+} \approx 3.6$, which means that the low-pressure regions in figure 7 that convect with about $6 \mathrm{u}_{\tau}$ originate close to the riblet crest and are likely created by the KelvinHelmholtz instability. Based on pressure fluctuations in the relatively short time interval of figure $7(b)\left(600 \nu / \mathrm{u}_{\tau}^{2}\right.$ compared to the total averaging time $\left.L_{t} \approx 65 \delta / u_{\tau}=25675 \nu / u_{\tau}^{2}\right)$, it appears that these Kelvin-Helmholtz rollers persist for times of roughly $100 \nu / u_{\tau}^{2}$ to $200 \nu / u_{\tau}^{2}$ before they break up and form anew. The faster structures that dominate the smooth-wall flow are only faintly visible over the riblets (e.g. marked by a short ...... around $t^{+} \approx 485$ and $x^{+} \approx 700$ in figure $7 b$ ).

\subsection{Evidence in spectral space}

In order to analyse the effect of Kelvin-Helmholtz rollers on momentum transfer, we focus on their contribution to Reynolds shear stress. Figure 8 shows premultiplied 2D 


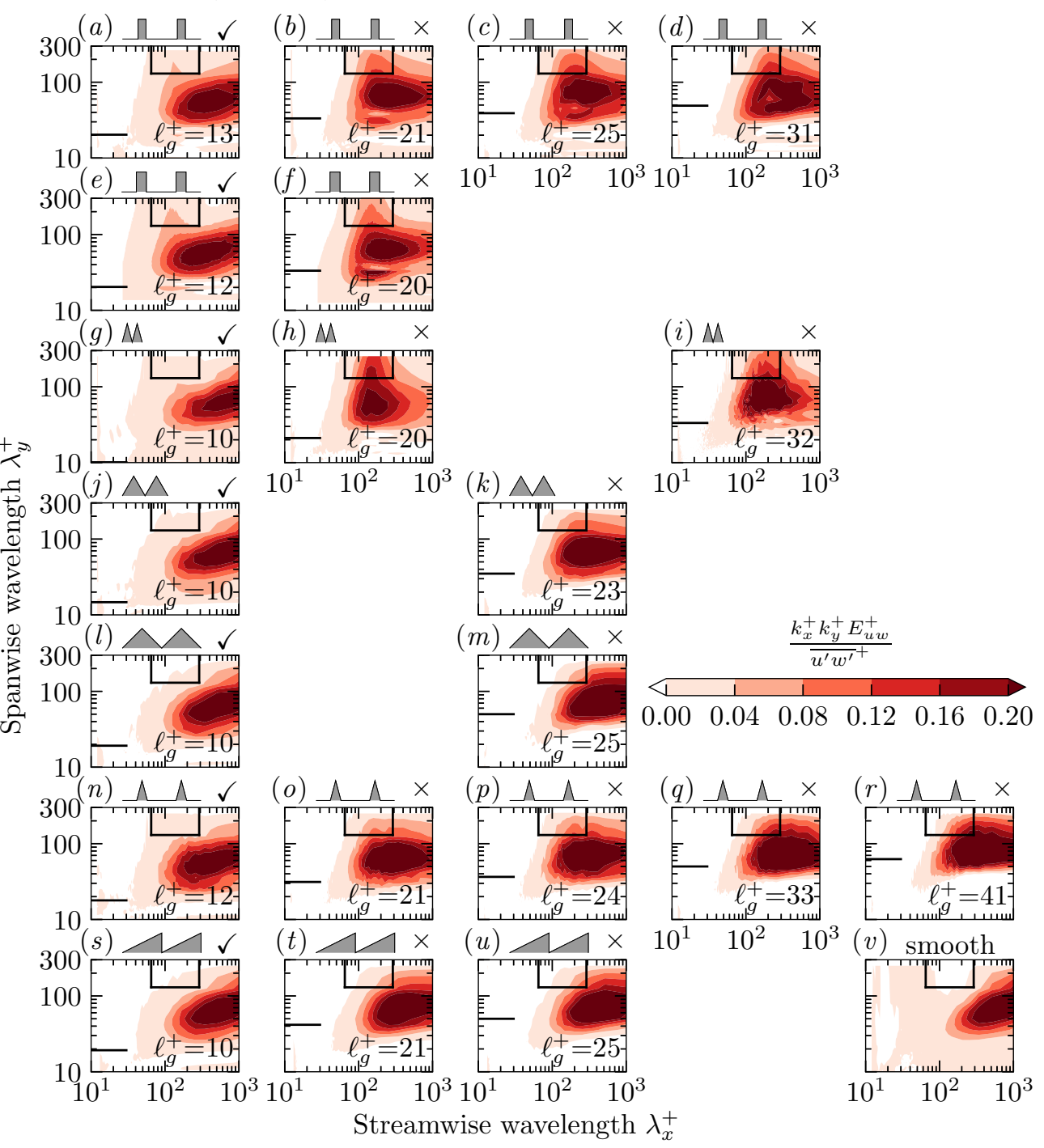

Figure 8. Premultiplied 2D co-spectra of Reynolds shear stress $k_{x}^{+} k_{y}^{+} E_{u w}^{+}$in the plane $3 \nu / \mathrm{u}_{\tau}$ above the riblet crest $(a-u)$ or smooth wall $(v)$. Horizontal lines on the left mark the riblet spacing $s^{+}$and open boxes near the top delimit the region of Kelvin-Helmholtz rollers $\left(65 \lesssim \lambda_{x}^{+} \lesssim 290, \lambda_{y}^{+} \gtrsim 130\right)$ according to García-Mayoral \& Jiménez $(2011 b)$. Normalisation: $\overline{u^{\prime} w^{\prime}}=\int_{0}^{\infty} \int_{0}^{\infty} E_{u w}^{+} \mathrm{d} \lambda_{x}^{+} \mathrm{d} \lambda_{y}^{+}$by considering that e.g. $E\left(\lambda_{x}, \lambda_{y}\right)+E\left(\lambda_{x},-\lambda_{y}\right)=E\left(-\lambda_{x}, \lambda_{y}\right)+E\left(-\lambda_{x},-\lambda_{y}\right)$. Cases 13L $(e)$ and 20L $(f)$ are for channel flow data from García-Mayoral \& Jiménez (2012). In the upper right, $(\checkmark)$ flags drag reducing and $(x)$ drag increasing cases.

spectra of Reynolds shear stress in a plane 3 viscous units above the riblet crests. Contour lines are normalised by ${\overline{u^{\prime} w^{\prime}}}^{+}$at that height to illustrate the distribution in spectral space as it changes with riblet size and shape. The normalisation excludes dispersive Reynolds stresses $\widetilde{\widetilde{u}}^{+}$, that are streamwise invariant for $2 \mathrm{D}$ riblets and thus located at $\left(\lambda_{x}^{+}, \lambda_{y}^{+}\right)=\left(\infty, s^{+}\right)$and the spanwise harmonics of that mode, which are not visible in figure 8 . The two smaller blade riblets, with a spacing-to-thickness ratio $s / t=5$, are of approximately the same size and shape as two of the blade riblets from García-Mayoral 
\& Jiménez $(2011 b, 2012)$ with $s / t=4$. The distributions of Reynolds stresses in spectral space, shown for the present and the reference cases in figure $8(a, b)$ and figure $8(e, f)$, respectively, match closely. The domains used by García-Mayoral \& Jiménez (2012) at $R e_{\tau} \approx 550$ are roughly 9 to 10 times wider in viscous units than our minimal-span channels at $R e_{\tau}=395$, but the close agreement in figure 8 demonstrates that the small domains correctly capture the resolved scales. Energy that would be at $\lambda_{y}^{+}>L_{y}^{+}$if the domains were larger instead accumulates in the infinite mode as shown by García-Mayoral \& Jiménez (2012) for full-span channels with varied spanwise extent.

Large blade and triangular riblets with $\alpha=30^{\circ}$ show spanwise coherent structures in visualizations of wall-shear stress (figure 6 ). For these cases, Reynolds shear stress close to the wall is amplified at large spanwise wavelengths and $\lambda_{x}^{+} \approx 180$ in figure $8(b, c, d, h, i)$. This is the same spectral region that García-Mayoral \& Jiménez (2011b) associate with KelvinHelmholtz rollers. They include streamwise wavelengths in the range $65 \lesssim \lambda_{x}^{+} \lesssim 290$ and large spanwise wavelengths $\lambda_{y}^{+} \gtrsim 130$, which we frame with a black box near the top of each spectrogram in figure 8 . The instability appears to be more pronounced over the sharp triangular riblets (figure $8 h, i$ ) than over the blades (figure $8 b, c, d$ ). The small riblets near the drag optimum of both shapes (figure $8 a, g$ ) have a weak peak in the same spectral region, suggesting that the instability exists but does not develop as strongly as for larger riblets, in agreement with García-Mayoral \& Jiménez (2011b). In general, drag reducing cases (marked $\checkmark$ ) have little energy in wavelengths related to the instability, much like the smooth wall flow at that height (figure $8 v$ ). Triangular riblets with $\alpha=60^{\circ}-90^{\circ}$ (figure $8 j-m, s-u$ ) and trapezoidal riblets (figure $8 n-r$ ) show little or no energy in the framed spectral regions associated with spanwise aligned rollers, regardless of their groove size. For the largest trapezoids TA50 and TA63 (figure 8q,r), some fluctuations related to the main peak from the near-wall cycle extend to large spanwise wavelengths, but their energy this close to the wall is low compared to the flow over triangular riblets with the same opening angle $\alpha=30^{\circ}$ or the blades.

The Reynolds stress associated with Kelvin-Helmholtz rollers can be estimated by integrating the corresponding part of the spectra in figure 8 at every wall-normal location. Parts of that spectral region are also affected by turbulence from the near-wall streaks and vortices, which are responsible for the main peak in the $2 \mathrm{D}$ spectra in figure 8 . As a conservative choice for the strength of Kelvin-Helmholtz rollers, and to exclude structures from the near-wall cycle, we henceforth only consider spanwise wavelengths $\lambda_{y}^{+} \gtrsim 250$ to be affected by the instability. In minimal-span channels with $L_{y}^{+} \approx 250$, this lower bound essentially reduces the integral to the first two spanwise modes, which correspond to $\lambda_{y}^{+}=\infty$ and $\lambda_{y}^{+} \approx 250$ respectively. Full-span channels resolve some of the wavelengths $\lambda_{y}^{+}>250$, whereas energy in our minimal-span channels at wavelengths $\lambda_{y}^{+}>L_{y}^{+} \approx 250$ accumulates in the $\lambda_{y}^{+}=\infty$ mode. Therefore, the small integration region in narrow domains captures all energy of fluctuations that are due to the Kelvin-Helmholtz instability. A more detailed analysis is given by Endrikat et al. (2020).

In figure 9 , spectra of Reynolds shear stress are integrated for the largest spanwise wavelengths $\left(\lambda_{y}^{+} \gtrsim 250\right)$ only and shown against distance from the riblet crest. Large spanwise-aligned structures that are compatible with a Kelvin-Helmholtz instability are clearly visible close to the crest of blade riblets (figure $9 a-f$ ) and sharp triangular riblets with $\alpha=30^{\circ}$ (figure $9 g-i$ ), but not over the other shapes investigated here. For trapezoidal and blunt triangular riblets, this portion of the spectrum is similar to that of smoothwall flow in figure $9(v)$. For the riblet cases that appear to support development of the instability, the peak in figure 9 seems to move upwards away from the crest and from the virtual origin as the riblet size, and thus the drag of the surface, increases. Reynolds stress related to quasi-streamwise vortices in the near-wall cycle on the other hand remains at 


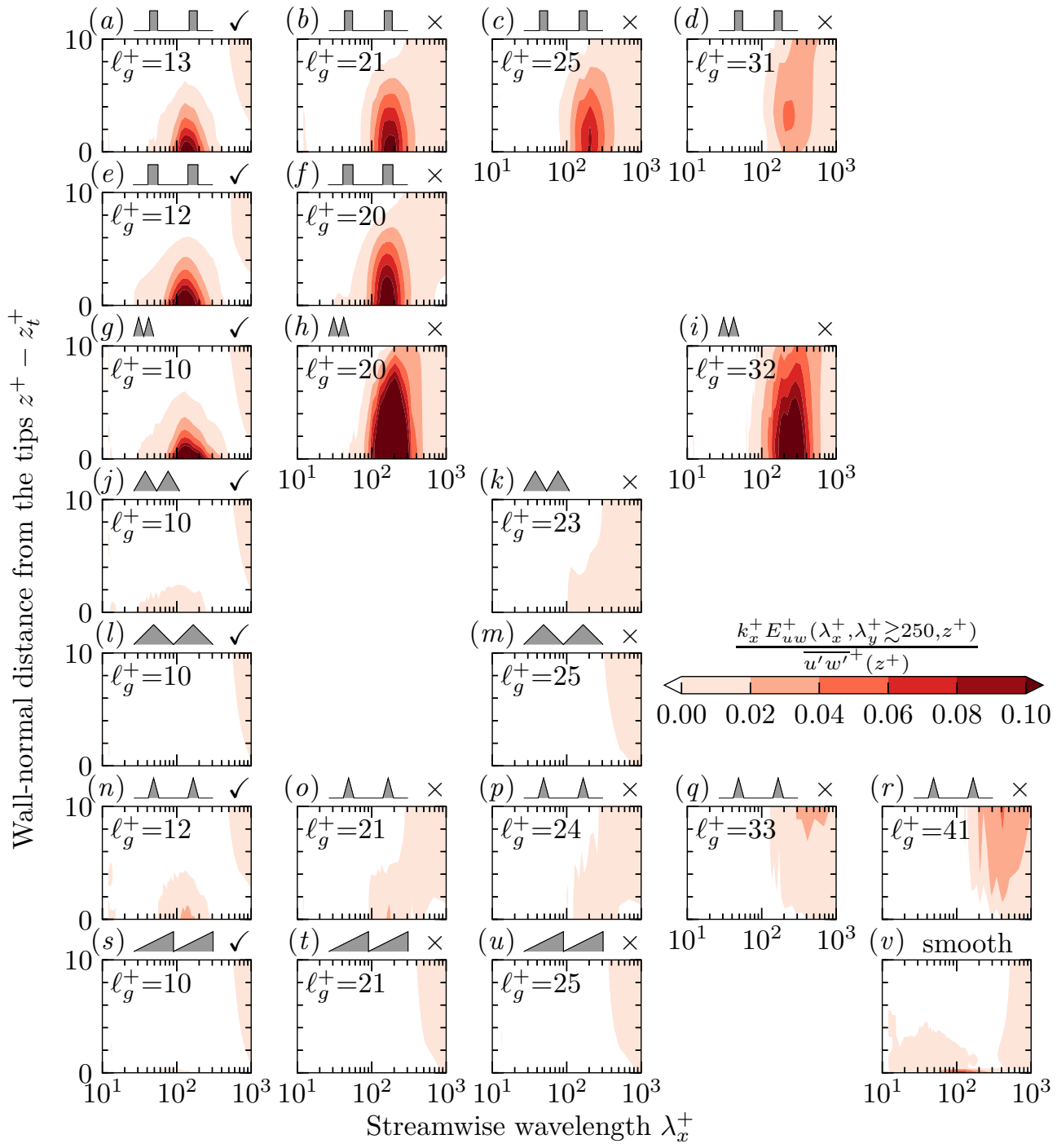

Figure 9. Premultiplied 1D co-spectra of Reynolds shear stress $k_{x}^{+} E_{u w}^{+}\left(\lambda_{y}^{+} \gtrsim 250\right)$ integrated only for large spanwise wavelengths. Same normalisation at every height as in figure 8 . Cases 13L $(e)$ and 20L $(f)$ are for channel flow data from García-Mayoral \& Jiménez (2012). In the upper right, $(\checkmark)$ flags drag reducing and $(\times)$ drag increasing cases.

about the same distance from the crest for all riblet sizes (figure $5 d$ ) as the virtual origin is found farther below the crest. The wall-normal location of Kelvin-Helmholtz rollers is not captured by the virtual origin, because they do not exist in the reference smoothwall flow and are therefore not part of the smooth-wall-like flow that we shift in the wall-normal direction to define the virtual origin $(\S 2.3)$.

The dominant $\lambda_{x}^{+}$of Kelvin-Helmholtz rollers seems to increase slightly with increasing riblet size (figure $9 a-d, g-i$ ). This trend was previously reported by Chavarin \& Luhar (2019) for a resolvent analysis of riblet flow and for plant canopies in experiments by Raupach et al. (1996) and based on a linear stability analysis by Sharma \& GarcíaMayoral (2020b). The range of streamwise wavelengths that are affected by the KelvinHelmholtz instability might therefore shift to higher values for very large riblets. However, 

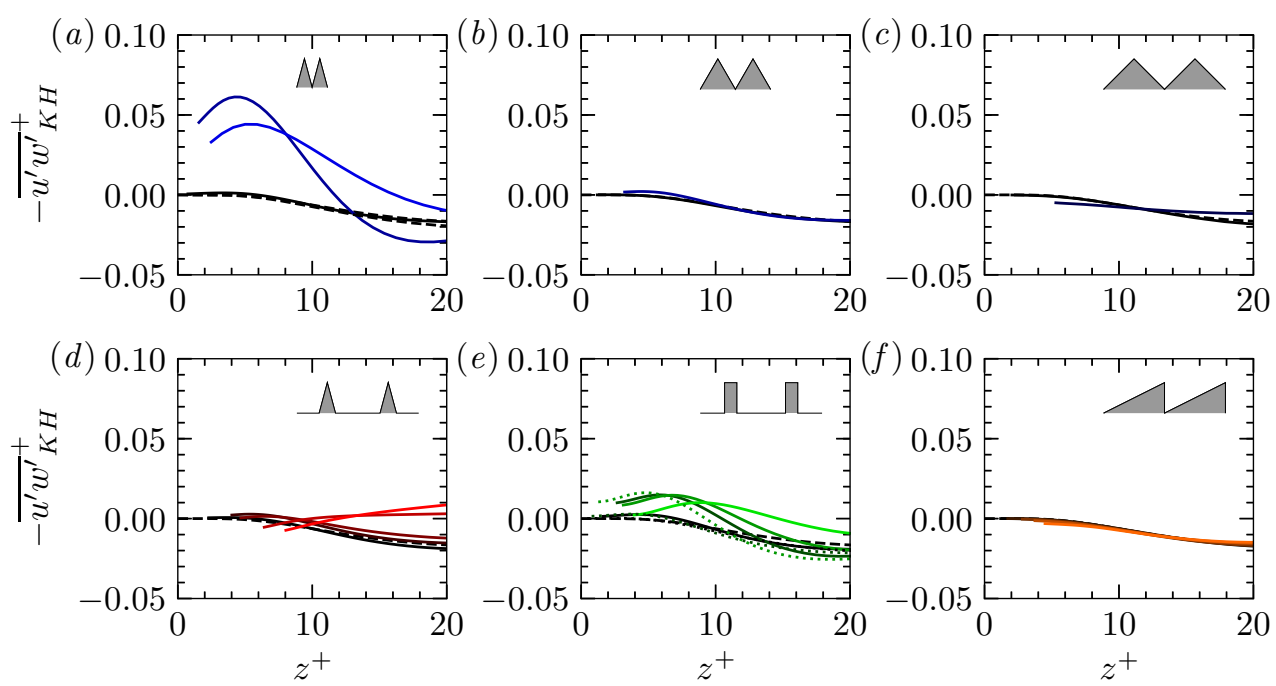

Figure 10. Profiles of Reynolds shear stress associated with Kelvin-Helmholtz rollers ${\overline{u^{\prime} w_{K H}^{\prime}}}_{K H}^{+}=\int_{250}^{\infty} \int_{65}^{290} E_{u w}^{+} d \lambda_{x}^{+} d \lambda_{y}^{+}$starting at the crest with lighter colours as riblet size increases. Smooth-wall profile is dashed. Additional smooth-wall profile in $(a)$ is at $R e_{\tau}=1000$ matching the largest (lightest) sharp triangular riblet case. Dotted profiles in $(e)$ are cases 07L, 13L and 20L from García-Mayoral \& Jiménez $(2012)$ at $R e_{\tau} \approx 550$.

for the present riblets we nevertheless consider fluctuations in the range $65 \lesssim \lambda_{x}^{+} \lesssim 290$, given by García-Mayoral \& Jiménez $(2011 b)$ for blade riblets up to $\ell_{g}^{+}=20$, to avoid including energy from the near-wall cycle at larger $\lambda_{x}^{+}$. After integrating over spanwise wavelengths affected by the instability, we now also integrate over these streamwise wavelengths, to obtain profiles of Reynolds stress associated with Kelvin-Helmholtz rollers. In other words, we split Reynolds shear stress at every height into

$$
{\overline{u^{\prime} w^{\prime}}}_{K H}^{+}\left(z^{+}\right)=\int_{250}^{\infty} \int_{65}^{290} E_{u w}^{+} \mathrm{d} \lambda_{x}^{+} \mathrm{d} \lambda_{y}^{+}
$$

due to the instability and a remainder.

Profiles of Reynolds shear stress associated with the Kelvin-Helmholtz instability are shown in figure 10 for the six riblet shapes and different sizes. Profiles for the large sharp triangular and blade riblets (figure $10 a, e$ ) have a peak below $z^{+} \approx 10$ (measured from the virtual origin), which is not seen in smooth-wall flow, and is therefore due to Kelvin-Helmholtz rollers. The flow over small drag reducing riblets of both shapes (dark lines) resembles that of the smooth wall as rollers are not supported by grooves with $\ell_{g}^{+} \lesssim 11$ (García-Mayoral \& Jiménez 2011b). Data for blade riblets by García-Mayoral \& Jiménez $(2012)$ at $R e_{\tau} \approx 550$ in full-span channels (dotted in figure $10 e$ ) show the same trend. Profiles for the blunt triangular riblets (figure 10b,c,f) are close to the smooth-wall profile, even for larger riblets, which we interpret as Kelvin-Helmholtz rollers not being present over these surfaces. The slanted triangular riblets in figure $10(f)$ are a particularly convincing example of riblets that do not alter Reynolds stresses in the spectral region associated with the Kelvin-Helmholtz instability, as the profiles for all riblet sizes are almost identical to that of the smooth wall. Profiles of $-{\overline{u^{\prime} w^{\prime}}}_{K H}^{+}$for the trapezoidal riblets (figure $10 d$ ) are also similar to the smooth-wall reference, except for the largest two cases (TA50, TA63) that increase drag substantially. For these two cases, $-{\overline{u^{\prime} w^{\prime}}}_{K H}^{+}$ increases monotonically with distance from the crest, which is not observed for riblets of 
traditional (near drag-reducing) size. At these very large riblet sizes $\left(s^{+}=50,63\right)$, the broad-band near-wall turbulence (strongest peak of Reynolds stress in figure 8) extends across a wide range of wavelengths and partly into the spectral region that is otherwise associated with the instability. Therefore, the two largest trapezoidal cases are excluded from the following analysis of the drag change due to Kelvin-Helmholtz rollers.

\section{Drag-change decomposition}

As discussed in the previous section, structures related to a shear-flow instability found over sharp triangular and blade riblets account for a significant portion of Reynolds shear stress close to the wall, while the flow over blunt triangular and trapezoidal riblets resembles more closely that of a smooth wall. The Reynolds shear stress transports streamwise momentum in the wall-normal direction and is therefore directly related to skin-friction drag experienced by a surface. We consider a momentum integral to decompose the drag-change in order to quantify if and how Kelvin-Helmholtz rollers affect the drag characteristics of a riblet surface. García-Mayoral \& Jiménez (2011b) used this concept to decompose the skin-friction coefficient and MacDonald et al. (2016) applied it instead to the Reynolds number independent $\Delta U^{+}$, which has since been a useful tool to gain insight into the drag characteristics of various surfaces (e.g. Jelly \& Busse 2018; Abderrahaman-Elena et al. 2019; Gómez-de-Segura \& García-Mayoral 2019). For our setup, $\Delta U^{+}$is decomposed as follows. The streamwise mean-momentum balance for turbulent channel flow above the riblet tips

$$
\frac{1}{\rho} \frac{\mathrm{d} P}{\mathrm{~d} x}=-\frac{1}{\delta} \frac{\tau_{w}}{\rho}=-\frac{\mathrm{d} \overline{u^{\prime} w^{\prime}}}{\mathrm{d} z}-\frac{\mathrm{d} \widetilde{\widetilde{u} \widetilde{w}}}{\mathrm{~d} z}+\nu \frac{\mathrm{d}^{2} U}{\mathrm{~d} z^{2}}
$$

is integrated in the wall-normal direction $z$ to obtain the viscous scaled total stress

$$
\frac{\delta^{++}-z^{+}}{\delta^{+}}=-{\overline{u^{\prime} w^{\prime}}}^{+}-\overline{\widetilde{u}}^{+}+\frac{\mathrm{d} U^{+}}{\mathrm{d} z^{+}}
$$

as the sum of Reynolds and viscous stresses. The discrepancy between both sides of (4.2), defined in (2.5), is given by $\varepsilon^{\prime+}$ in table 1 . Our simulations are set up such that $\delta$ measures the distance between the riblet mean-height and the top of the open channel (figure $2 b$ ) to match the cross-sectional areas of smooth-wall and riblet channels. Therefore, the total stress, extrapolated below the riblet crest, reaches $\tau_{w}$ at the mean-height. The effective half-channel height, i.e. the distance between the virtual origin and top of the domain is $\delta^{\prime}$. The total stress on the left-hand side of (4.2) is described using both $\delta^{+}$ and $\delta^{\prime+}$, because $\tau_{w}$ is not measured at the virtual origin and therefore $\delta \neq \delta^{\prime}$ (table 1 ). After integrating again between the riblet tips at $z_{t}^{+}$and the height up to which data are representative of full-span channel flow $z_{c}^{+}$, we find an expression for the mean streamwise velocity at $z_{c}^{+}$,

$$
U^{+}\left(z_{c}^{+}\right)=\int_{z_{t}^{+}}^{z_{c}^{+}} \frac{\delta^{\prime+}-z^{+}}{\delta^{+}} \mathrm{d} z^{+}+\int_{z_{t}^{+}}^{z_{c}^{+}}{\overline{u^{\prime} w^{\prime}}}^{+}+\overline{\widetilde{u}}^{+} \mathrm{d} z^{+}+U^{+}\left(z_{t}^{+}\right),
$$

as a function of integrated stress profiles. Finally, we subtract (4.3) from the same equation for a smooth wall (denoted by a subscript $s$ ) that is positioned at the height of the virtual origin $(\S 2.3)$ to obtain an expression for the velocity shift that measures the 

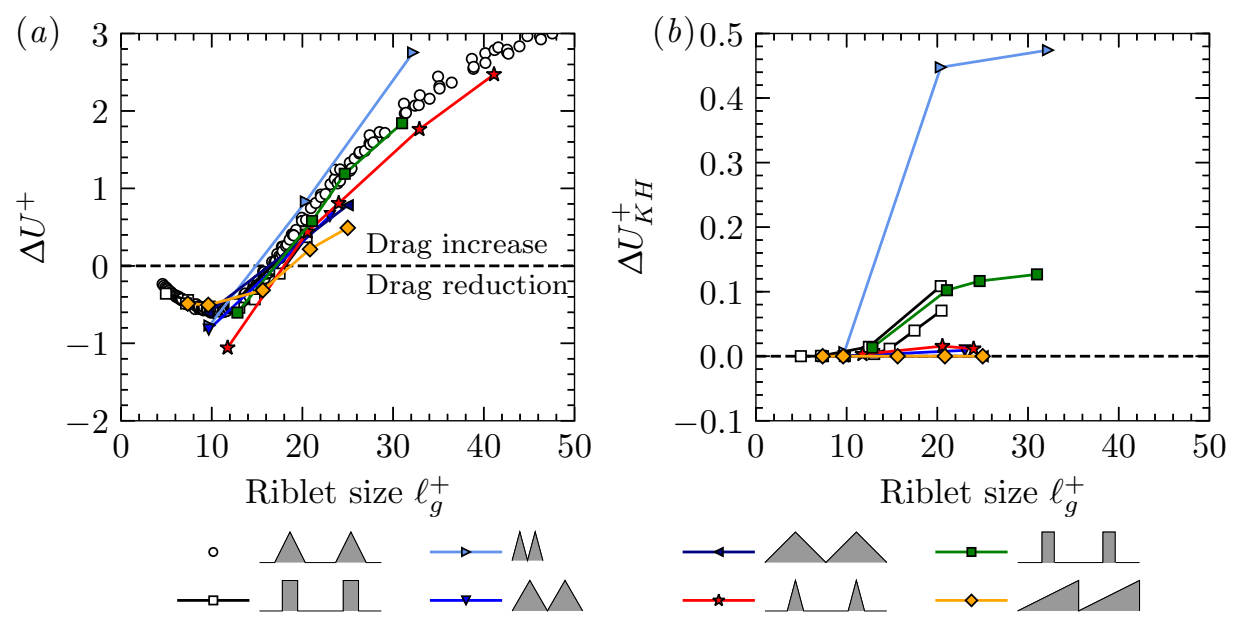

FiguRE 11. (a) Drag-change compared to a smooth wall $\Delta U^{+}$. (b) Contribution of Kelvin-Helmholtz rollers to $\Delta U^{+}$. Reference data $\bigcirc$ at $R e_{\tau} \approx 150-2270$ experimentally obtained by Deyn et al. (2019) and $\square$ at $R e_{\tau} \approx 180-550$ from DNSs by García-Mayoral \& Jiménez (2012).

drag change

$$
\begin{array}{cl}
\Delta U^{+}\left(z_{c}^{+}\right)= & U_{s}^{+}-U^{+}=\Delta U_{t}^{+}+\underbrace{\Delta U_{u w}^{+}}_{\Delta U_{K H}^{+}+\Delta U_{R}^{+}}, \\
\text {where } \quad \Delta U_{t}^{+}=U_{s}^{+}\left(z_{t}^{+}\right)-U^{+}\left(z_{t}^{+}\right) \\
\Delta U_{u w}^{+}=\int_{z_{t}^{+}}^{z_{c}^{+}} \frac{\delta_{s}^{+}-z^{+}}{\delta_{s}^{+}}-\frac{\delta^{\prime+}-z^{+}}{\delta^{+}} \mathrm{d} z^{+}+\int_{z_{t}^{+}}^{z_{c}^{+}}{\overline{u^{\prime} w^{\prime}}}_{s}^{+}-{\overline{u^{\prime} w^{\prime}}}^{+}-\overline{\widetilde{u}}^{+} \mathrm{d} z^{+} \\
\Delta U_{K H}^{+}=\int_{z_{t}^{+}}^{z_{c}^{+}}{\overline{u^{\prime} w^{\prime}}}_{K H, s}^{+}-{\overline{u^{\prime} w^{\prime}}}_{K H}^{+} \mathrm{d} z^{+} .
\end{array}
$$

The term $\Delta U_{u w}^{+}$includes the difference of total stresses, which is a measure of the $R e_{\tau}$ mismatch after adjusting the origin and not an effect of riblets on the drag-change, as discussed by Gómez-de-Segura \& García-Mayoral (2019) in the context of permeable surfaces. However, we only consider the Reynolds stresses ${\overline{u^{\prime} w^{\prime}}}^{+}$and split them in spectral space according to (3.1) into those due to Kelvin-Helmholtz rollers ${\overline{u^{\prime} w^{\prime}}}_{K H}^{+}$ and a remainder (subscript $R$ ). We therefore follow the approach by García-Mayoral \& Jiménez $(2011 b)$, except with a more conservative integration over $\lambda_{y}^{+} \gtrsim 250$ instead of $\lambda_{y}^{+} \gtrsim 50$. In figures 9 and 10 we see that the effect of Kelvin-Helmholtz rollers is mostly limited to the first 15-20 viscous units above the crest. Similarly, García-Mayoral \& Jiménez $(2011 b)$ observe them within $20 \nu / \mathrm{u}_{\tau}$ to $30 \nu / \mathrm{u}_{\tau}$ from the tips of blade riblets and integrate the difference in the stresses ${\overline{u^{\prime} w^{\prime}}}_{K H, s}^{+}-{\overline{u^{\prime} w^{\prime}}}_{K H}^{+}$up to $z^{+}=35$. For the present cases however, the peak in figure $10(a, e)$ shifts to slightly higher $z^{+}$with increasing riblet size. Therefore, instead of integrating up to a fixed height, we consider a difference in the stresses ${\overline{u^{\prime} w^{\prime}}}_{K H, s}^{+}-{\overline{u^{\prime} w^{\prime}}}_{K H}^{+}$only where the riblet-wall profile of $-{\overline{u^{\prime} w^{\prime}}}_{K H}^{+}$is positive in figure 10. After this adjustment, the remaining difference is integrated up to $z_{c}^{+}$as per (4.7) to find the drag-change due solely to Kelvin-Helmholtz rollers $\Delta U_{K H}^{+}$.

The total drag-change $\Delta U^{+}$and the contribution to it by Kelvin-Helmholtz rollers are shown in figure 11 for all riblet cases. The flow over blunt triangular and trapezoidal riblets resembles that over a smooth wall in the spectral region associated with KelvinHelmholtz rollers (figure 10). Therefore, the integrated difference $\Delta U_{K H}^{+}$in figure $11(b)$ 
amounts to negligible drag changes. For sharp triangular and blade riblets, the instability contributes significantly to the drag-change in agreement with data by García-Mayoral \& Jiménez (2012).

Nevertheless, $\Delta U_{K H}^{+}<\Delta U^{+}$which means that even without the drag-change due to the Kelvin-Helmholtz instability, large riblets increase drag above smooth-wall values. This suggests that flow mechanisms other than those related to the Kelvin-Helmholtz instability, like dispersive stresses from secondary flows (Goldstein \& Tuan 1998), might also contribute to the drag-increase relative to a smooth wall for large riblets. Given that the occurrence of drag-increasing Kelvin-Helmholtz rollers depends strongly on the riblet shape, other possible mechanisms of drag increase (Choi et al. 1993; Goldstein \& Tuan 1998) might likewise depend on the exact groove geometry.

\section{Indicators for the development of Kelvin-Helmholtz rollers over riblets}

Having observed a dependence of the drag penalty of Kelvin-Helmholtz rollers on the riblet shape in figure $11(b)$, we now explore indicators that describe which riblet surfaces trigger the Kelvin-Helmholtz instability. The strength of the Kelvin-Helmholtz instability in wall-bounded flows depends on the wall-normal permeability of the surface (e.g. Jiménez et al. 2001; García-Mayoral \& Jiménez 2011b; Gómez-de-Segura \& GarcíaMayoral 2019) and on the mixing layer around the roughness tips as observed for plant canopies (e.g. Raupach et al. 1996; Nepf et al. 2007; Sharma \& García-Mayoral 2020b). Here we characterise both separately for riblets.

\subsection{How the wall-normal permeability varies with riblet size}

Riblet grooves allow streamwise flow below the riblet crest, akin to porous substrates that are preferentially permeable in $x$. For these surfaces, the appearance of KelvinHelmholtz rollers is fully described by the wall-normal permeability at the interface between flow and substrate (Gómez-de-Segura et al. 2018a). Jiménez et al. (2001) describe a permeable boundary by relating wall-normal velocity to fluctuations of pressure through the porosity coefficient $\beta=-\rho w / p^{\prime}$, that has the dimensions of an inverse velocity. In the context of riblets, we therefore use $\beta$ evaluated in the plane at the crest as a measure of the wall-normal permeability that the groove provides. Following Gómez-de-Segura et al. (2018b), we calculate the magnitude of the porosity coefficient $\left|\beta^{+}\right|\left(\lambda_{x}^{+}, \lambda_{y}^{+}, t^{+}\right)=\left|\hat{w}^{+}\right| /\left|\hat{p}^{+}\right|$, where $\hat{\cdot}$ denotes Fourier coefficients and retain a dependence on wavelengths and time. Only large spanwise wavelengths $\lambda_{y}^{+} \gtrsim 250$ are affected by the Kelvin-Helmholtz instability $(\S 3.2)$ and figure 12 shows the probability of the porosity coefficient for these wavelengths $\left|\beta^{+}\right|_{\lambda_{y}^{+} \gtrsim 250}$ taking a certain value. The impermeability condition of the smooth wall prohibits all wall-normal motion in figure $12(t)$, in contrast to riblets of any size and shape. In flow over riblets, $\left|\beta^{+}\right|_{\lambda_{y}^{+} \approx 250}$ depends strongly on the streamwise wavelength with the highest values around $\lambda_{x}^{+} \approx 20-40$. The porosity coefficient of small, drag-reducing riblets $(\checkmark)$ has a high probability of falling into a narrow range of $\left|\beta^{+}\right|_{\lambda_{y}^{+} \geq 250}$, i.e. it is fairly stationary at given $\lambda_{x}^{+}$. For drag-increasing cases $(\times),\left|\beta^{+}\right|_{\lambda_{y}^{+} \geq 250}$ takes higher values than over small riblets, because wall-normal velocity fluctuations are greater above large grooves. Furthermore, $\left|\beta^{+}\right|_{\lambda_{y}^{+} \gtrsim 250}$ fluctuates more in time than for small riblets, i.e. the observed occurrences are spread out across many bins in figure 12 and the contours are lighter. However, the drag-increasing cases that support Kelvin-Helmholtz rollers, based on the drag-change decomposition in figure $11(b)$, reduce the range of values that $\left|\beta^{+}\right|_{\lambda_{y}^{+} \gtrsim 250}$ takes for those wavelengths 


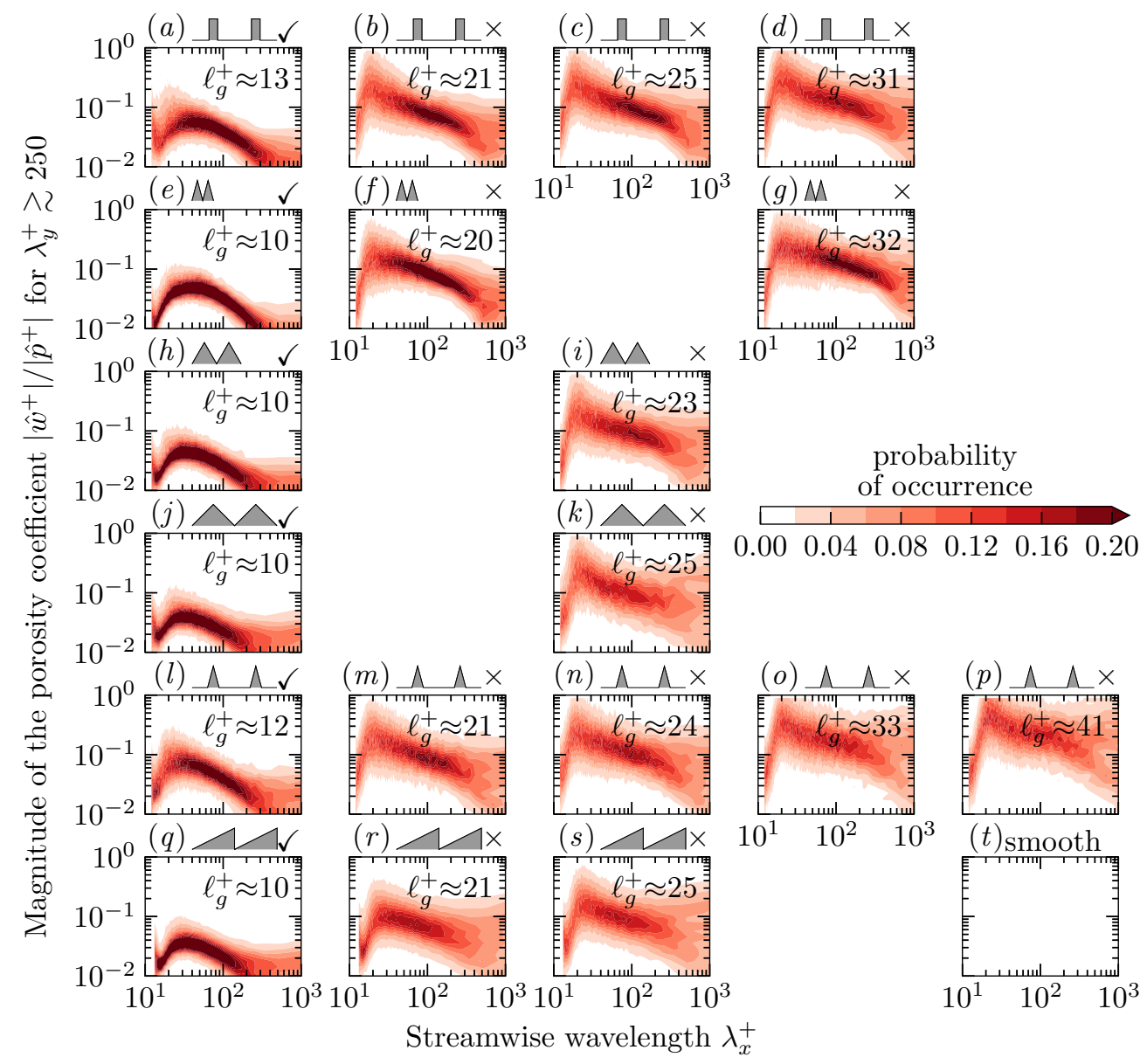

FIGURE 12. Ratio between wall-normal velocity and pressure fluctuations for large spanwise wavelengths $\lambda_{y}^{+} \gtrsim 250$ at the riblet crest. Colours represent the probability of $\left|\beta^{+}\right|_{\lambda_{y}^{+} \gtrsim 250}$ falling into each of 30 logarithmically spaced bins between $10^{-3}$ and 1 at a given $\lambda_{x}^{+}$. Ticks on the vertical axis mark the bins. On the right, $\checkmark$ mark drag-reducing and $\times$ drag-increasing cases.

that are affected by the instability $\left(65 \lesssim \lambda_{x}^{+} \lesssim 290\right)$. Coherent Kelvin-Helmholtz rollers therefore appear to reduce the randomness of fluctuations of $\left|\beta^{+}\right|_{\lambda_{y}^{+} \gtrsim 250}$ in time, which is here visible as concentrated dark spots in figure 12. This is most noticeable for the triangular riblets with $\alpha=30^{\circ}$ (figure $12 f, g$ ) and blades (figure $12 b, c, d$ ), but also to a lesser extent for the triangular riblets with $\alpha=60^{\circ}$ (figure 12i) and mid-sized trapezoids (figure $12 m, n$ ). Over larger riblets without Kelvin-Helmholtz rollers on the other hand (figure $12 k, o, p, r, s),\left|\beta^{+}\right|_{\lambda_{y}^{+} \gtrsim 250}$ is more randomly distributed in time and not fixed by coherent motions.

We find the porosity coefficient averaged in time and over wavenumbers $k_{x}$ and $k_{y}$ affected by the Kelvin-Helmholtz instability

$$
{\overline{\mid \beta^{+}}}_{K H}=\frac{1}{\frac{2 \pi}{250}} \frac{1}{\frac{2 \pi}{65}-\frac{2 \pi}{290}} \int_{0}^{\frac{2 \pi}{250}} \int_{\frac{2 \pi}{290}}^{\frac{2 \pi}{65}} \overline{\left|\hat{w}^{+}\right| /\left|\hat{p}^{+}\right|} \mathrm{d} k_{x}^{+} \mathrm{d} k_{y}^{+}
$$

for all cases and observe an almost linear increase with riblet size in figure 13. Curves for the different shapes collapse when shown against the groove size $\ell_{g}^{+}$rather than 

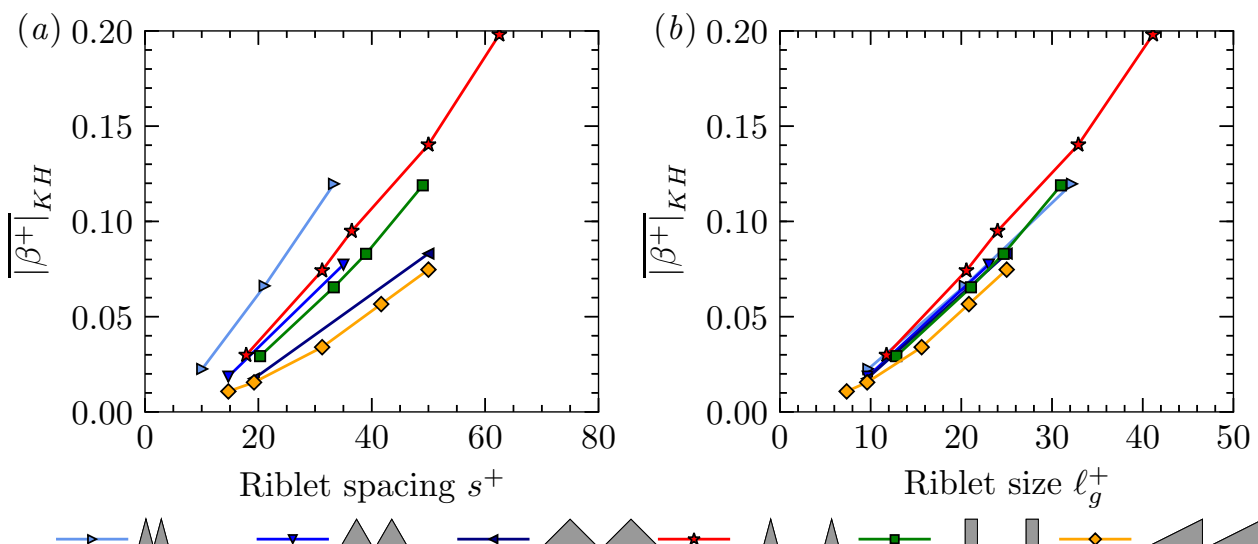

FIGURE 13. Time averaged porosity coefficient at the riblet crest integrated over wavelengths that may be affected by Kelvin-Helmholtz rollers ${\overline{\mid \beta^{+}}}_{K H}$.

the riblet spacing, presumably because the volume available below the crest determines the response in terms of $w$ to a given pressure disturbance. Therefore, the geometrical parameter $\ell_{g}^{+}$correlates with the wall-normal permeability of fully open grooves. Indeed, García-Mayoral \& Jiménez $(2011 b)$ observe rollers over blade riblets with $\ell_{g}^{+} \gtrsim 11$ (also figure $11 b$ ), and attribute it to the sufficiently permeable interface between riblet grooves and the overlying flow. They propose a model that accounts for this permeability through a viscous approximation for the flow in the groove and also find a linear increase with $\ell_{g}^{+}$, that explains why $\Delta U_{K H}^{+} \approx 0$ for small riblets with $\ell_{g}^{+} \lesssim 11$ in figure $11(b)$. Likewise for porous surfaces, Kelvin-Helmholtz rollers appear if the (Darcy) wall-normal permeability exceeds a threshold, $\sqrt{K_{z}^{+}} \gtrsim 0.4$, as observed in DNSs (Gómez-de-Segura \& García-Mayoral 2019) and predicted by resolvent analysis (Chavarin et al. 2020). Furthermore, for superhydrophobic microgrooves, which can be considered riblets with free-slip grooves, Rastegari \& Akhavan (2018) also observe a dependence of the KelvinHelmholtz instability on the mean depth of the grooves. Reynolds stress spectra at $z^{+}-z_{t}^{+} \approx 5$ above these free-slip grooves in their figure $7(c-f, i)$ show that energy in the spectral region of Kelvin-Helmholtz rollers increases with $\ell_{g}^{+}$. However, even the free-slip grooves with a small $\ell_{g}^{+} \approx 6$ by Rastegari \& Akhavan (2018) exhibit KelvinHelmholtz rollers, presumably because the slip condition allows the flow to easily move along the groove, which in turn leads to large wall-normal permeability of the plane at the crest, despite the low $\ell_{g}^{+}$. This is consistent with the model of García-Mayoral \& Jiménez $(2011 b)$ for the flow within the groove and its effect on the Kelvin-Helmholtz instability. In appendix $\S \mathrm{A}$, we show results from a numerical experiment in which the formation of Kelvin-Helmholtz rollers is shown to be suppressed when the riblet grooves are replaced by impermeable and flat $\left(\ell_{g}^{+}=0\right)$ free-slip strips at the crest.

The increasing permeability of the plane at the riblet crest in figure 13(b) barely depends on the riblet shape, and it therefore does not explain the results from our drag-change decomposition $(\S 4)$ that four of the six riblet shapes do not support strong Kelvin-Helmholtz rollers regardless of $\ell_{g}^{+}$. We therefore additionally consider shear in the mixing layer as a second indicative parameter that is affected by both the riblet shape and size. 


\subsection{How riblets affect shear in the mixing layer}

Kelvin-Helmholtz rollers can develop in a mixing layer that forms between slow flow in the riblet groove and a faster stream above, as first suggested by Raupach et al. (1996) for plant canopies and later assumed by García-Mayoral \& Jiménez (2011b) for riblets. This mixing layer is susceptible to the Kelvin-Helmholtz instability, because the profile of mean-steamwise velocity has an inflection point at the canopy or riblet tips, which is a necessary condition for instability in free shear flows (Rayleigh 1879). Above streamwise porous surfaces however, the wall-normal permeability alone describes the appearance of Kelvin-Helmholtz rollers (Gómez-de-Segura et al. 2018a; Chavarin et al. 2020) and the linear stability analysis by García-Mayoral \& Jiménez (2011b) predicts the instability as a result of wall-normal permeability of riblets for non-inflectional velocity profiles. Nevertheless, many of our large (drag-increasing) riblets do not experience significant drag due to the instability (§4) despite their wall-normal permeability (figure 13b). Therefore, we will now consider shear strength in the mixing layer as a second parameter to describe flow conditions that generate Kelvin-Helmholtz rollers above riblets. In this section we first follow the characterisation of the mixing layer from previous literature, by investigating the shear length scale $L_{s}$ (Raupach et al. 1996) and a mixing-length model (Poggi et al. 2004). However, the complete description of the mixing layer for riblets emerges when we additionally consider the distribution of wall-shear stress across the riblet height.

\subsubsection{Shear length scale}

At the height of the inflection point, Raupach et al. (1996) measure shear in the mixing layer relative to the mean velocity by defining the shear length scale $L_{s} \equiv U_{t} /(\mathrm{d} U / \mathrm{d} z)$, which decreases with increasing shear. For plant canopies, various studies (e.g. Raupach et al. 1996; Dunn et al. 1996; Finnigan 2000; Poggi et al. 2004; Coceal \& Belcher 2004) consider the velocity profile after normalising $U / U_{t}$ and $z / k$. This way, the vertical velocity gradient at the crest can be rewritten as $k / L_{s}$, i.e. as the shear length scale relative to the canopy height. Based on the drag-decomposition in figure $11(b)$, we would expect the strongest shear for the triangular riblets with $\alpha=30^{\circ}$, followed by the blades and weaker shear for the remaining four shapes. Indeed, the velocity profiles for the sharp triangular riblets are steepest (figure 14a) and the gradient at the crest, where the instability originates, is strongest (figure 14c). We also observe in figure 14(c), that shear in the mixing layer appears to become weaker with increasing riblet size, which suggests that it might be too weak to support Kelvin-Helmholtz rollers for very large riblets. Furthermore, it is conceivable that Kelvin-Helmholtz rollers need to perceive spanwise homogeneous shear to develop, which widely spaced riblet tips might not provide. In DNS of small (drag-reducing) riblets, turbulent flow in large parts of the groove is dominated by viscosity. We can therefore describe the near-wall flow field in the limit $\ell_{g}^{+} \sim 0$ by streamwise shear-driven Stokes flow $\nabla^{2} u=0$ with a fixed velocity at the top and no-slip walls on the bottom. The Stokes flows differ from the turbulent flow of larger riblets, because they lack turbulent motions including Kelvin-Helmholtz rollers and secondary flows in the riblet cross-section. However, in agreement with García-Mayoral \& Jiménez $(2011 b)$, superficially spanwise averaged velocity profiles inside the smallest riblet grooves of each geometry (figure 14a) closely resemble the Stokes flow solution (figure 14b). Above the crest, the Stokes flow is no longer representative of the turbulent solution and the profiles are linear, because the flow is driven by shear rather than a pressure gradient. Directly at the crest however, where we characterise the mixing layer, the Stokes-flow simulations correctly predict a strong velocity gradient for the triangular riblets with 

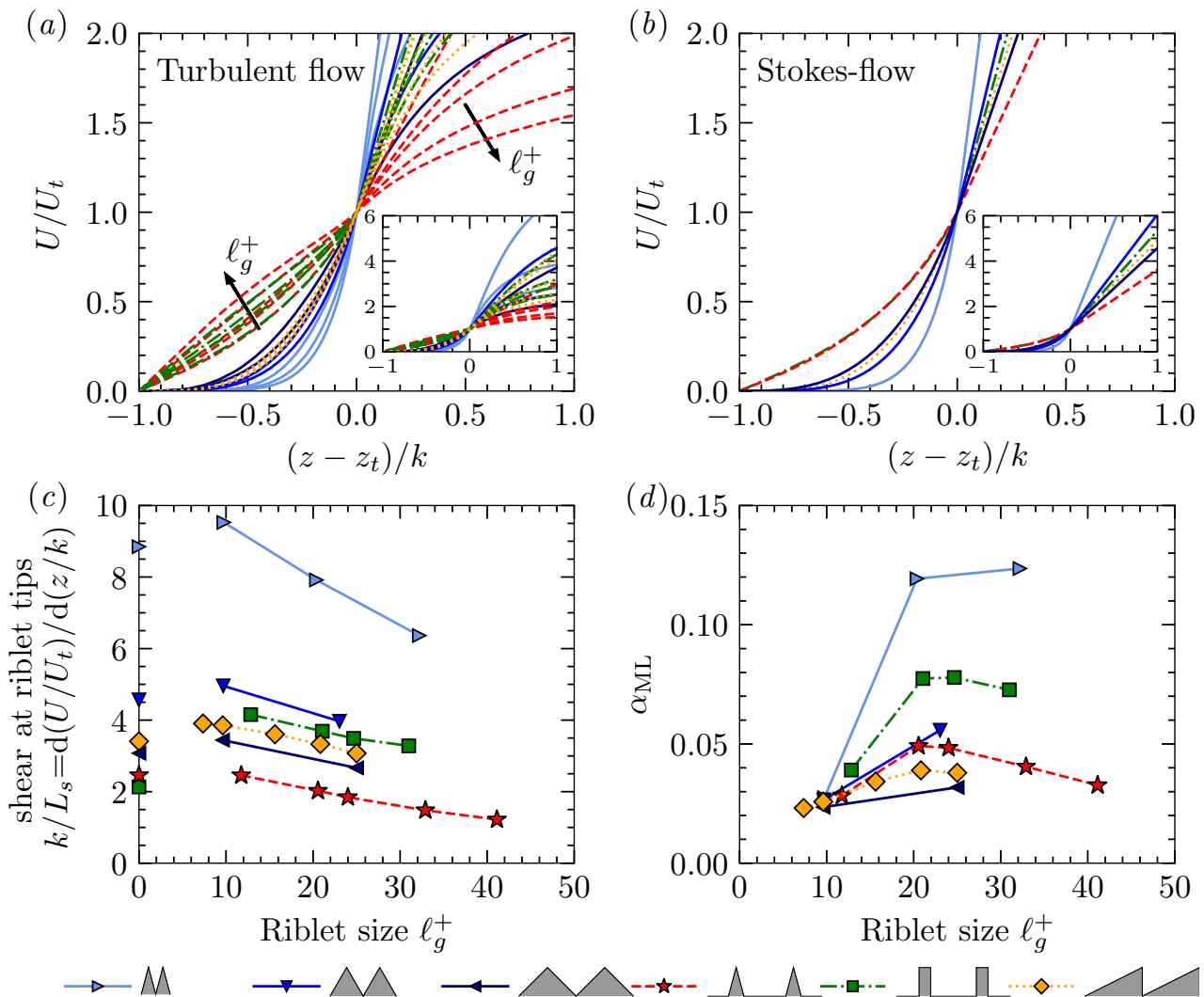

FiguRE 14. Superficially averaged velocity profiles in and above the groove normalised by the riblet height $k$ and velocity at the tips. For $(a)$ turbulent flow and $(b) 2 \mathrm{D}$ Stokes-flow. Insets show a larger velocity range. $(c)$ Velocity gradient at the riblet crest expressed using the shear length scale $L_{s}^{+}=U_{t}^{+} /\left(\mathrm{d} U^{+} / \mathrm{d} z^{+}\right)_{t}$ relative to the groove-depth $k^{+}$(Raupach et al. 1996) with Stokes-flow values for $\ell_{g}^{+} \sim 0$. $(d)$ Contribution of the mixing-layer length to the effective total mixing length at the riblet crest based on the model by Poggi et al. (2004).

$\alpha=30^{\circ}$ (shown in figure $14(c)$ at $\ell_{g}^{+}=0$ ) and a more gentle velocity increase for the other shapes. The Stokes-flow gradient for the blades is low and thus indicative of a weak mixing layer, even though they support Kelvin-Helmholtz rollers in turbulent flow (§3 and §4). Furthermore, the shear-strength in turbulent flow over the blades in figure $14(c)$ is similar to that of the slanted triangular riblets for which the instability is absent. This suggests that the velocity gradient at the riblet crest, in Stokes and turbulent flow alike, does not fully describe the mixing layer that can give rise to Kelvin-Helmholtz rollers. We therefore additionally consider the mixing-length model proposed by Poggi et al. (2004) to describe the mixing layer at the tips of plant canopies.

\subsubsection{Mixing-length model}

Poggi et al. (2004) compare the mixing length due to the Kelvin-Helmholtz instability $\ell_{\mathrm{ML}}^{+}=L_{s}^{+} \equiv U_{t}^{+} /\left(\mathrm{d} U^{+} / \mathrm{d} z^{+}\right)$to that of turbulent eddies that are likewise found over a smooth wall $\ell_{\mathrm{BL}}^{+}$. The tips of plant canopies by Poggi et al. (2004), where the instability develops, are in the log-layer at heights hundreds of viscous units and about $0.08 \delta$ above the virtual origin. At those heights above a smooth wall, the mixing length is $\ell_{\mathrm{BL}}^{+}=\kappa z^{+}$, with the von-Kármán constant $\kappa=0.4$. For riblets, the inflection point of the velocity 
profile is also at the tips (• in figure 4 ), which is however much closer to the virtual origin $(\S 2.3)$. For the present cases, the inflection point is at heights $\ell_{T}^{+} \approx 0.8-8.0$. Above a smooth wall at those heights, in the viscous sublayer or buffer-layer, viscous effects reduce $\ell_{\mathrm{BL}}^{+}$, which van Driest (1956) accounts for by multiplying the mixing length with the damping term $\left(1-e^{-z^{+} / 26}\right)$. Here, the constant 26 was determined empirically to recover a measured smooth-wall velocity profile (van Driest 1956). After including that damping term to account for viscous effects, the mixing-length scale representing smoothwall flow at the height of the riblet crest $z^{+}=\ell_{T}^{+}$is given by $\ell_{\mathrm{BL}}^{+}=\kappa \ell_{T}^{+}\left(1-e^{-\ell_{T}^{+} / 26}\right)$.

Poggi et al. (2004) propose that inside the mixing layer around the canopy tips, the mixing lengths of the instability and of turbulent eddies can be superimposed with the weighting parameter $\alpha_{\mathrm{ML}}$ to describe the effective mixing length in that region $\ell_{\mathrm{eff}}^{+}=\left(1-\alpha_{\mathrm{ML}}\right) \ell_{\mathrm{BL}}^{+}+\alpha_{\mathrm{ML}} \ell_{\mathrm{ML}}^{+}$. By modelling the Reynolds stress and the eddydiffusivity using the velocity gradient, they can describe the effective mixing length as

$\ell_{\text {eff }}^{+}=\sqrt{-\overline{u^{\prime} w^{\prime}}} /\left(\mathrm{d} U^{+} / \mathrm{d} z^{+}\right)$. We measure $\ell_{\text {eff }}^{+}$at the riblet crest and solve for the parameter $\alpha_{\mathrm{ML}}$ that describes the relative contribution of the mixing-layer length (Kelvin-Helmholtz rollers) and of background turbulence $\ell_{\mathrm{BL}}^{+}$to the net wall-normal momentum transfer.

The sparsest canopy investigated by Poggi et al. (2004) has $\alpha_{\mathrm{ML}} \approx 0$ and does not create a mixing layer. The instability starts to emerge for the denser cases with $\alpha_{\mathrm{ML}} \approx 0.05,0.25$ and $\alpha_{\mathrm{ML}}$ appears to asymptote towards 0.5 for the densest canopies. The triangular riblets with opening angle $\alpha=30^{\circ}$ have the highest values of $\alpha_{\mathrm{ML}}$ in figure $14(d)$, because the Kelvin-Helmholtz instability contributes significantly to the momentum transfer, as seen in $\S 4$. The blades have reduced $\alpha_{\mathrm{ML}}$, that is however noticeably higher than for the remaining riblet shapes that do not support the Kelvin-Helmholtz instability. Drag reducing riblets of all shapes have small $\alpha_{\mathrm{ML}}$, and therefore weak mixing layers, in agreement with the drag-decomposition in $\S 4$. Interestingly, the differences between riblet shapes in $\alpha_{\mathrm{ML}}$ (figure 14d) can be traced back to the slip length $\ell_{T}^{+}$and the slip (crest) velocity $U_{t}^{+}(\S \mathrm{B})$.

\subsubsection{Distribution of wall-shear stress}

In order to relate the riblet geometry to the strength of shear in the mixing layer, we consider the distribution of wall-shear stress across the riblet, by integrating it below every height to obtain profiles $\tau_{w, z}$ (figure $15 a$ ). The analysis is motivated by the observation that plant canopies create an inflectional velocity profile with a mixing layer that supports Kelvin-Helmholtz rollers if the drag exerted by plants is large compared to the bed-drag on the ground (White \& Nepf 2007). Similarly for riblets in figure 15(a), a steep curve in the tip region indicates a significant momentum absorption over that wallnormal distance, which creates a strong mixing layer. The flat tips of blade riblets with a finite thickness have high shear stress such that the flow over blades loses a significant proportion of momentum to the tip region, which creates a discontinuity of wall-shear stress at the riblet crest. The other riblet shapes all have a pointed crest and therefore a smooth distribution of $\tau_{w, z}$. Nevertheless, the triangular riblets with $\alpha=30^{\circ}$ experience most wall-shear stress near the tips, which explains low groove velocities in figure $14(a)$. On the other hand, trapezoidal riblets for example, with the same opening angle $\alpha=30^{\circ}$, have a more balanced distribution of $\tau_{w, z}$ across the height owing to their larger tip spacing at the same $\ell_{g}^{+}$and the flat bottom of the groove. For these and other riblet shapes with somewhat evenly distributed wall-shear stress in figure 15(a), streamwise momentum penetrates farther into the groove, which appears to weaken the mixing layer at the riblet tips to a point where it no longer supports the Kelvin-Helmholtz instability. Stokes-flow in figure 15(b) shows the same trend between riblet shapes, because even 

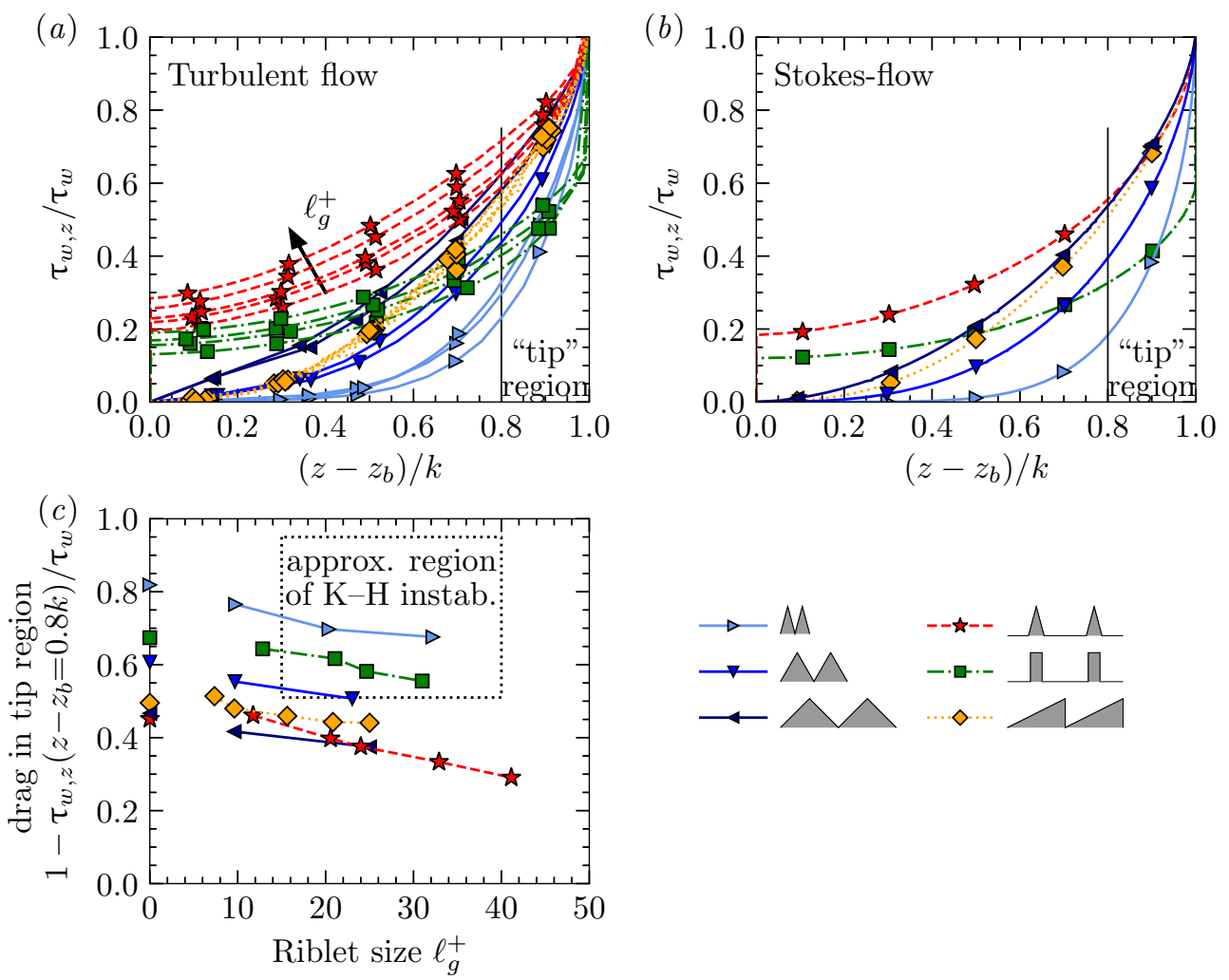

FiguRE 15. Wall-shear stress (drag per unit plan area) accumulated from below given heights $z, \tau_{w, z}=\int_{L_{t}} \int_{A(z)} \nu \rho(\partial u / \partial n) \mathrm{d} A \mathrm{~d} t /\left(L_{x} L_{y} L_{t}\right)$ relative to the total wall-shear stress $\tau_{w}=\int_{L_{t}} \int_{A\left(z_{t}\right)} \nu \rho(\partial u / \partial n) \mathrm{d} A \mathrm{~d} t /\left(L_{x} L_{y} L_{t}\right)$, where $n$ is the locally wall-perpendicular direction and $A(z)$ the wetted wall-surface below height $z$. The domain extents $L_{x}, L_{y}$ and time-averaging intervals $L_{t}$ are given in table 1 . For $(a)$ DNSs of turbulent flow and (b) 2D Stokes-flows. (c) Fraction of wall-shear stress that acts on only the top $20 \%$ of the riblets (threshold is marked by vertical line in $a, b)$ with Stokes-flow values that are valid for $\ell_{g}^{+} \sim 0$.

the turbulent flow at these riblet sizes is dominated by viscosity in large parts of the groove. Our idea that high drag at the tips promotes Kelvin-Helmholtz rollers is further supported by the DNSs of flow over superhydrophobic free-slip microgrooves by Rastegari \& Akhavan (2018). Based on spectra of Reynolds shear stress, Kelvin-Helmholtz rollers only develop over the free-slip grooves (their figure $7 c-f, i$ ), that are set up to absorb all momentum at the tips. The no-slip riblet versions of the same microgrooves in their figure $7(l-o, r)$ lack pronounced Kelvin-Helmholtz rollers, because a significant portion of the wall-shear stress acts well below the crest.

In order to measure the effect of high wall-shear at the riblet crest, we pick a threshold at $\left(z-z_{b}\right) / k=0.8$ to integrate the wall-shear stress only over the tip region and show that portion of the total $\tau_{w}$ in figure $15(c)$. As expected from the drag-decomposition, triangular riblets with $\alpha=30^{\circ}$ and blades experience most wall-shear stress near the riblet crest. The distribution of wall-shear stress across the riblet height correlates reasonably well with the existence and strength of Kelvin-Helmholtz rollers for all six riblet shapes including the blades, as only high values in the tip-region appear to lead to strong mixing layers that support the instability. Even though the threshold for the tip region 
at $\left(z-z_{b}\right) / k=0.8$ is arbitrary, figure $15(a)$ shows that any threshold between roughly $0.75 \lesssim\left(z-z_{b}\right) / k \lesssim 0.95$ leads to qualitatively matching results.

The approximate region of riblets that support Kelvin-Helmholtz rollers in figure 15(c) is delimited by the two parameters discussed in this section. Wall-shear stress in the tip region has to be roughly above the lower bound based on the drag-change decomposition for this data set (figure $11 b$ ) and there should be a maximum above which fluctuations are damped by high resistance to streamwise flow in the groove (Nepf et al. 2007). On the horizontal axis in figure 15(c), small grooves with low wall-normal permeability prevent fluctuations (figure 13 along with figure $11 b$ ) and very large riblets seem to have weak shear in the mixing layers (figure 14). Wall-shear stress from Stokes-flow for $\ell_{g}^{+} \sim 0$ in figure 15(c) shows the same trend we observe in turbulent flow. Therefore, the strength of Kelvin-Helmholtz rollers over other riblet shapes could be estimated by taking $\ell_{g}^{+}$as an indicator for the wall-normal permeability and then comparing the Stokes-flow wall-shear stress in the tip region to values from the present data set, for which we found $\Delta U_{K H}^{+}$ (figure 11b). The absence of strong drag-increasing Kelvin-Helmholtz rollers outside of the approximate region in figure $15(c)$ does not mean that these riblet shapes provide improved drag reduction compared to riblets that support the instability (figure $11 a$ ). For example, dispersive Reynolds stresses associated with secondary cross flows (Goldstein \& Tuan 1998) can contribute to drag for large riblets, including potentially alongside Kelvin-Helmholtz rollers.

\section{Conclusion}

Kelvin-Helmholtz rollers have been known to develop in the flow above plant canopies (e.g. Raupach et al. 1996; Nepf et al. 2007; Sharma \& García-Mayoral 2020b), permeable surfaces (e.g. Jiménez et al. 2001; Breugem et al. 2006; Gómez-de-Segura \& GarcíaMayoral 2019) and riblets (García-Mayoral \& Jiménez 2011b), because these boundaries can all create a mixing layer with an inflectional velocity profile and provide sufficient wall-normal permeability for the instability to develop. We conducted direct numerical simulations of turbulent flow over six riblet shapes and various viscous-scaled sizes to investigate conditions for the appearance of the Kelvin-Helmholtz instability. We decomposed the drag change $\Delta U^{+}$between riblet surfaces and a smooth wall to extract the drag penalty due to Kelvin-Helmholtz rollers $\Delta U_{K H}^{+}$. In agreement with results by García-Mayoral \& Jiménez $(2011 b), \Delta U_{K H}^{+} \approx 0$ for small riblets near the drag optimum, because the impermeability condition of the wall obstructs the roll-up of coherent structures. The instability contributes significantly to the drag-change for larger, dragincreasing triangular riblets with opening angle $\alpha=30^{\circ}$ and blade riblets with a spacingto-thickness ratio $s / t=5$. However, trapezoidal riblets with $\alpha=30^{\circ}$ and triangular riblets with $\alpha=60^{\circ}$ to $\alpha=90^{\circ}$ have $\Delta U_{K H}^{+} \approx 0$ regardless of their viscous-scaled groove size. We therefore proposed two parameters that together describe the occurrence of the KelvinHelmholtz instability in turbulent flow over riblets.

First, Kelvin-Helmholtz rollers may only develop if the viscous-scaled groove volume is sufficiently large to provide the necessary wall-normal permeability of the plane at the riblet crest, as demonstrated for blade riblets by García-Mayoral \& Jiménez (2011b). We illustrated this by averaging the porosity coefficient, i.e. the ratio of fluctuations of wallnormal velocity and pressure, across wavelengths affected by the instability. This measure of the wall-normal permeability increases linearly with the groove size $\ell_{g}^{+}$regardless of the riblet shape. Therefore, the first parameter illustrates why the instability is absent for small $\left(\ell_{g}^{+} \lesssim 11\right)$ riblets with fully open grooves (García-Mayoral \& Jiménez 2011b). However, this parameter alone is insufficient to explain why only certain riblet shapes 
sustain the Kelvin-Helmholtz instability at larger $\ell_{g}^{+}$and correspondingly high wallnormal permeability.

The second parameter describes the strength of shear in the mixing layer around the inflection point at the crest from which the Kelvin-Helmholtz instability develops. We first considered the velocity gradient relative to the riblet height, which is strongest for the triangular riblets with $\alpha=30^{\circ}$, but it does not fully explain why the blade riblets support Kelvin-Helmholtz rollers (figure 14c). However, a mixing-length model based on that by Poggi et al. (2004) appears to capture the relative contribution of Kelvin-Helmholtz rollers to turbulence for all considered riblet shapes (figure $14 d$ ). In order to arrive at a possibly more predictive and intuitive description of the mixing layer, we further considered the distribution of wall-shear stress across the riblet height and found that Kelvin-Helmholtz rollers only develop if the riblet tips absorb a large proportion of the momentum to create high shear in the neighbourhood of the inflection point. The Kelvin-Helmholtz instability is therefore absent for groove shapes that allow momentum to descend towards the bottom, like the blunt triangular or trapezoidal riblets we investigated. Wall-shear stress in the tip region appears to correlate more robustly with the occurrence of Kelvin-Helmholtz rollers for the six riblet shapes than the velocity gradient in the inflection point. Furthermore, qualitative distributions of wall-shear stress from 2D Stokes-flow calculations show the same trend across riblet shapes. Together with the geometrical parameter $\ell_{g}^{+}$that describes the wall-normal permeability, the Stokes-flow solution gauges the ability of these riblet shapes to support Kelvin-Helmholtz rollers.

The absorption of momentum in the tip region and the wall-normal permeability are generally connected, but adjusting the riblet shape has the potential to change one more than the other. As a result, four out of the six riblet shapes do not support strong Kelvin-Helmholtz rollers.

\section{Acknowledgements}

The authors gratefully acknowledge the support of the Australian Research Council Discovery Project DP170102595. This research is undertaken with the assistance of resources from NCI that is supported by the Australian Government and from Pawsey that is funded by the Australian Government and the Government of Western Australia. D. Modesti was partially funded by Ermenegildo Zegna, through the "EZ Founder's Scholarship".

Declaration of Interests: The authors report no conflict of interest.

\section{Appendix A. An impermeable boundary suppresses Kelvin-Helmholtz rollers}

The necessity of wall-normal permeability for the development of Kelvin-Helmholtz rollers has been discussed by Jiménez et al. (2001); García-Mayoral \& Jiménez (2011b); Gómez-de-Segura et al. (2018a); Gómez-de-Segura \& García-Mayoral (2019) based on stability analysis and DNSs. Nevertheless, here we demonstrate through comparison of the blade riblet case BL49 to an impermeable counterpart BS49, that Kelvin-Helmholtz rollers do not develop if the plane at the riblet crest is impermeable. The simulation BS49 has the same computational box, Reynolds number and mesh spacing as the blade riblet case BL49 (table 1), except that the riblet grooves are replaced by impermeable $(w=0)$ and non-deformable free-slip strips at the height of the riblet crest (following the setup of Martell, Perot \& Rothstein 2009).

At a height of 5 viscous units above the crest, where Kelvin-Helmholtz rollers may 

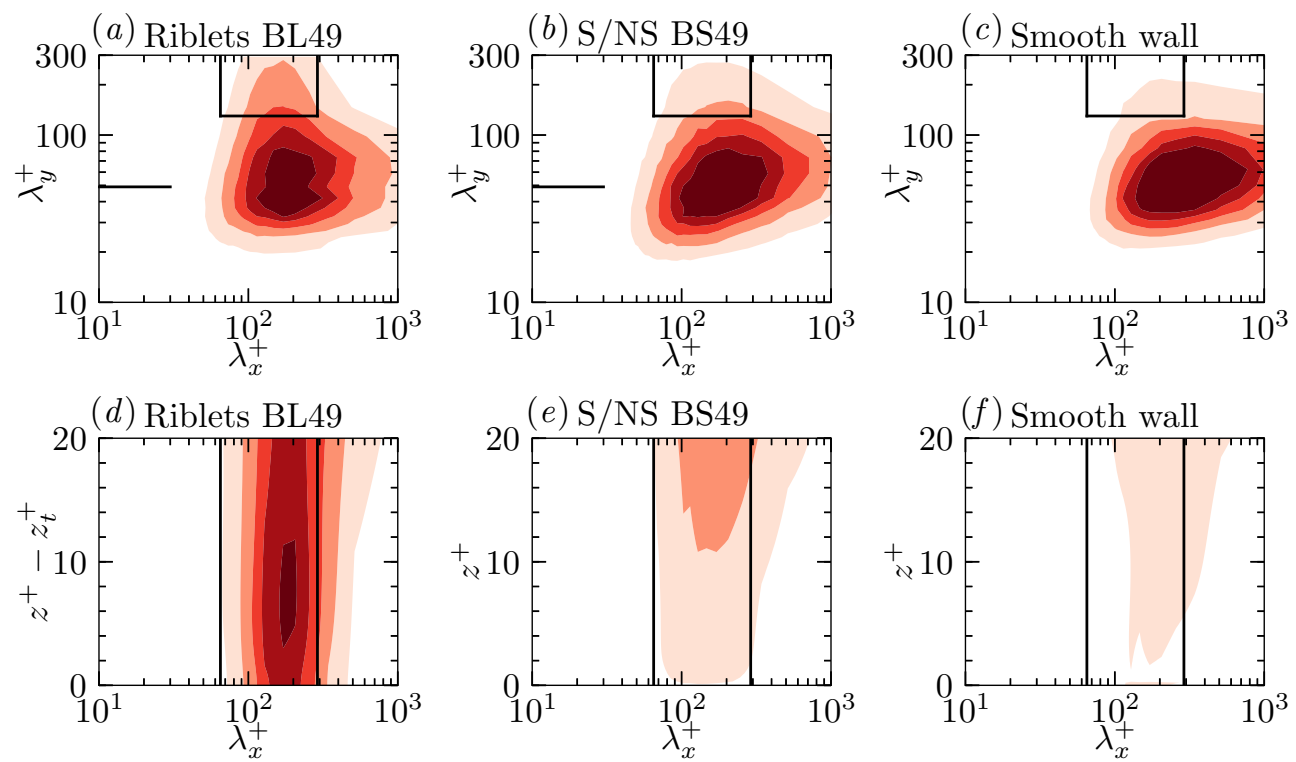

Figure 16. Premultiplied spectra of wall-normal velocity in the flow above blade riblets BL49 $(a, d)$, blades with free-slip strips instead of grooves BS49 $(b, e)$ and a uniform no-slip smooth wall $(c, f)$, normalised by the respective variance at each height. The $2 \mathrm{D}$ spectra $(a-c)$ are taken at a height of 5 viscous units (above the riblet crest) with 5 contour levels from light to dark in the range $[0.04,0.2] \overline{w^{\prime} w^{\prime}}$. The $1 \mathrm{D}$ spectra $(d-f)$ are only for large spanwise wavelengths $\lambda_{y}^{+} \gtrsim 250$ with 5 contour levels from light to dark in the range $[0.02,0.1]{\overline{w^{\prime} w^{\prime}}}^{+}$. The spectral region of Kelvin-Helmholtz rollers is framed by black lines $\left(65 \lesssim \lambda_{x}^{+} \lesssim 290, \lambda_{y}^{+} \gtrsim 130\right.$, as given by García-Mayoral \& Jiménez 2011b).

develop, the contours of the wall-normal velocity spectrum for the blade riblets (figure 16a) extend farther into the region of Kelvin-Helmholtz rollers than for the slip/noslip surface (figure 16b) and least for the uniform no-slip smooth wall (figure 16c). The large spanwise wavelengths $\left(\lambda_{y}^{+} \gtrsim 250\right)$ that may be affected by the Kelvin-Helmholtz instability in figure $16(d-f)$, carry far more energy over the blade riblets than over the slip/no-slip surface. Nevertheless, wall-normal velocity at the large spanwise wavelengths is slightly stronger over the slip/no-slip wall compared to the uniform no-slip smooth wall. Importantly though, these fluctuations do not extend down close to the surface as they do above the riblet. The permeable plane at the riblet crest allows for $w \neq 0$, which stability analysis shows to be critical for the development of Kelvin-Helmholtz rollers (Jiménez et al. 2001; García-Mayoral \& Jiménez 2011b, cf. mode shapes in their figure $19 c-e)$. Consequently, only the riblet flow in figure $16(d)$ has a distinct and isolated peak at $z^{+}-z_{t}^{+} \lesssim 12$ and $\lambda_{x}^{+} \approx 180$, i.e. in the region of Kelvin-Helmholtz rollers (§3.2). Decomposing the drag-change as in $\S 4$ shows that the slip/no-slip surface has a drag penalty from wavelengths in the region of Kelvin-Helmholtz rollers $\Delta U_{K H}^{+}=1.7 \times 10^{-5} \approx 0$, whereas for the blade riblets the penalty is $\Delta U_{K H}^{+} \approx 0.13$.

Overall, the spectral analysis of the impermeable (i.e. $\ell_{g}^{+}=0$ ) case BS49 supports our conclusions in $\S 5$ on Kelvin-Helmholtz rollers: shear forces the Kelvin-Helmholtz instability, but impermeability suppresses it. In figure 15(c), the slip/no-slip case BS49 with full momentum absorption at the riblet tips and $\ell_{g}^{+}=0$ would appear in the top left corner, outside of the region of Kelvin-Helmholtz rollers. 

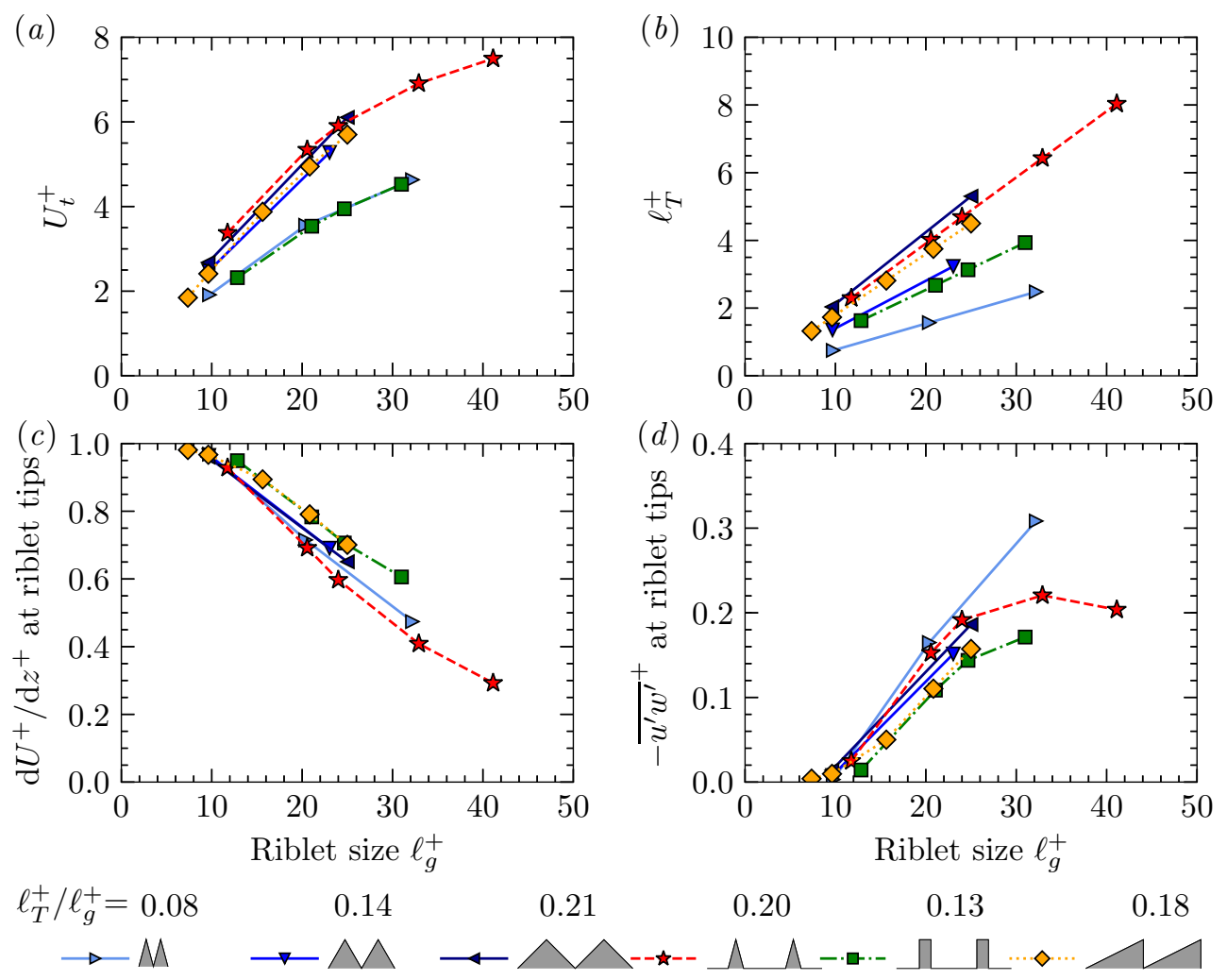

Figure 17. Terms at the riblet tips, that make up the smooth-wall mixing length $\ell_{\mathrm{BL}}^{+}=\kappa \ell_{T}^{+}\left(1-e^{-\ell_{T}^{+} / 26}\right)$, the mixing length due to the Kelvin-Helmholtz instability $\ell_{\mathrm{ML}}^{+}=L_{s}^{+}=U_{t}^{+} /\left(\mathrm{d} U^{+} / \mathrm{d} z^{+}\right)$and the combined effective mixing length $\ell_{\mathrm{eff}}^{+}=\sqrt{-\overline{{u^{\prime} w^{\prime}}^{+}}} /\left(\mathrm{d} U^{+} / \mathrm{d} z^{+}\right)$, following the model by Poggi et al. (2004) in $\S 5.2 .2$. The slip velocity at the riblet tips $U_{t}^{+}(a)$ is marked by $(\bullet)$ in the profiles of figure 4 . The slip length $\ell_{T}^{+}(b, \S 2.3)$ measures the distance between the virtual origin and the riblet crest.

\section{Appendix B. Flow at the riblet tips}

In the viscous regime of small riblet sizes, the slip velocity at the riblet tips $U_{t}^{+}$and the slip length $\ell_{T}^{+}$describe the drag-change $\Delta U^{+} \approx \ell_{T}^{+}-\ell_{U}^{+}$, because the streamwise protrusion height $\ell_{U}^{+} \approx U_{t}^{+}$(Luchini (1996) and $\S 1$ ). It appears that in the turbulent regime, the influence of the Kelvin-Helmholtz instability on the drag-change is qualitatively noticeable through the same two parameters: for cases with strong Kelvin-Helmholtz rollers, $U_{t}^{+}$and $\ell_{T}^{+}$are particularly low at a given $\ell_{g}^{+}$(figure $17(a, b)$ and ratio $\ell_{T}^{+} / \ell_{g}^{+}$in the legend), i.e. the virtual origin is close to the riblet crest. This has implications for the mixing length model ( $\S 5.2 .2$ based on that by Poggi et al. 2004), that compares the mixing length of Kelvin-Helmholtz rollers to that of turbulent eddies based on the slip length $\ell_{T}^{+}$and three quantities measured at the riblet crest: $U_{t}^{+}, \mathrm{d} U^{+} / \mathrm{d} z^{+}$and $\overline{u^{\prime} w^{\prime}}$. The last two vary as a function of $\ell_{g}^{+}$that is roughly the same for all considered riblet shapes (figure $17 c, d)$. Therefore, the value of $\alpha_{\mathrm{ML}}$ in the mixing length model, that indicates the presence of Kelvin-Helmholtz rollers in figure $14(d)$, is predominantly influenced by $\ell_{T}^{+}$and $U_{t}^{+}$. 


\section{REFERENCES}

Abderrahaman-Elena, N., Fairhall, C. T. \& García-Mayoral, R. 2019 Modulation of near-wall turbulence in the transitionally rough regime. J. Fluid Mech. 865, 1042-1071.

Bechert, D., Bruse, M., Hage, W., van der Hoeven, J. \& Hoppe, G. 1997 Experiments on drag-reducing surfaces and their optimization with an adjustable geometry. J. Fluid Mech. 338, 59-87.

Bilinsky, H. 2019 Direct contactless microfabrication of 3D riblets: Improved capability and metrology. AIAA Scitech 2019 Forum (AIAA Paper 2019-1626).

Breugem, W. P., Boersma, B. J. \& Uittenbogandd, R. E. 2006 The influence of wall permeability on turbulent channel flow. J. Fluid Mech. 562, 35-72.

Chan, L., MacDonald, M., Chung, D., Hutchins, N. \& Ooi, A. 2018 Secondary motion in turbulent pipe flow with three-dimensional roughness. J. Fluid Mech. 854, 5-33.

Chavarin, A., Gómez-De-Segura, G., García-Mayoral, R. \& Luhar, M. 2020 Resolventbased predictions for turbulent flow over anisotropic permeable substrates, arXiv: 2006.01378.

Chavarin, A. \& Luhar, M. 2019 Resolvent analysis for turbulent channel flow with riblets. AIAA J. 58 (2), 589-599.

Choi, H., Moin, P. \& Kim, J. 1993 Direct numerical simulation of turbulent flow over riblets. J. Fluid Mech. 255, 503-539.

Chu, D. C. \& Karniadakis, G. E. 1993 A direct numerical simulation of laminar and turbulent flow over riblet-mounted surfaces. J. Fluid Mech. 250, 1-42.

Chung, D., Chan, L., MacDonald, M., Hutchins, N. \& Ooi, A. 2015 A fast direct numerical simulation method for characterising hydraulic roughness. J. Fluid Mech. 773, 418-431.

Clauser, F. H. 1956 The turbulent boundary layer. Advances in App. Mech. 4, 1-51.

Coceal, O. \& Belcher, S. E. 2004 A canopy model of mean winds through urban areas. Quart. J. Royal Meteorological Soc. 130 (599), 1349-1372.

Deyn, L., Coppini, L., Hehner, M., Kriegseis, J., Gatti, D., Forooghi, P. \& FrohnAPfeL, B. 2019 Riblets in fully developed turbulent channel flow - an experimental campaign. EDRFCM (March 26-29 2019, Bad Herrenalb, Germany).

Drazin, P. G. \& Reid, W. H. 2004 Hydrodynamic Stability, 2nd edn. Cambridge Mathematical Library.

van Driest, E. R. 1956 On turbulent flow near a wall. J. Aeron. Sci. 23 (11), 1007-1011.

Dunn, C., Lopez, F. \& Garcia, M. 1996 Mean flow and turbulence in a laboratory channel with simulated vegetation. Civil Engineering Studies hydraulic engineering series (51), $0-162$.

Endrikat, S., Modesti, D., MacDonald, M., García-Mayoral, R., Hutchins, N. \& Chung, D. 2020 Direct numerical simulations of turbulent flow over various riblet shapes in minimal-span channels. Flow Turb. Comb. pp. 1-29.

Finnigan, J. 2000 Turbulence in plant canopies. Annu. Rev. Fluid Mech. 32 (1), 519-571.

Flores, O. \& JimÉnez, J. 2010 Hierarchy of minimal flow units in the logarithmic layer. Phys. Fluids 22 (7), 071704.

García-Mayoral, R., Gómez-De-Segura, G. \& Fairhall, C. T. 2019 The control of nearwall turbulence through surface texturing. Fluid Dyn. Res. 51 (1), 011410.

García-Mayoral, R. \& Jiménez, J. 2011a Drag reduction by riblets. Phil. Trans. R. Soc. A 369 (1940), 1412-1427.

García-Mayoral, R. \& JimÉnez, J. $2011 b$ Hydrodynamic stability and breakdown of the viscous regime over riblets. J. Fluid Mech. 678, 317-347.

García-Mayoral, R. \& JimÉnez, J. 2012 Scaling of turbulent structures in riblet channels up to $R e_{\tau} \approx 550$. Phys. Fluids 24 (10), 105101.

Goldstein, D. \& Tuan, T. C. 1998 Secondary flow induced by riblets. J. Fluid Mech. 363, $115-151$.

Gómez-De-Segura, G. \& García-Mayoral, R. 2019 Turbulent drag reduction by anisotropic permeable substrates - analysis and direct numerical simulations. J. Fluid Mech. 875, $124-172$.

Gómez-de-Segura, G., Sharma, A. \& García-Mayoral, R. 2018a Turbulent drag reduction using anisotropic permeable substrates. Flow Turb. Comb. 100 (4), 995-1014. 
Gómez-De-Segura, G., Sharma, A. \& García-Mayoral, R. $2018 b$ Virtual origins in turbulent flows over complex surfaces. Center for Turbulence Research Proceedings of the Summer Program 2018 (3), 277-286.

Ham, F., Mattsson, K. \& Iaccarino, G. 2006 Accurate and stable finite volume operators for unstructured flow solvers. Center for Turbulence Research, Stanford University/NASA Ames, Annual Research Briefs pp. 243-261.

Ham, F., Mattsson, K., Iaccarino, G. \& Moin, P. 2007 Towards time-stable and accurate LES on unstructured grids. In Complex Effects in Large Eddy Simulations, pp. 235-249. Springer Berlin Heidelberg.

HwAng, Y. 2013 Near-wall turbulent fluctuations in the absence of wide outer motions. J. Fluid Mech. 723, 264-288.

Iwamoto, K., Suzuki, Y. \& KAsagi, N. 2002 Reynolds number effect on wall turbulence: toward effective feedback control. Int. J. Heat Fluid Flow 23 (5), 678-689.

Jelly, T. \& Busse, A. 2018 Reynolds and dispersive shear stress contributions above highly skewed roughness. J. Fluid Mech. 852, 710-724.

Jiménez, J. \& Moin, P. 1991 The minimal flow unit in near-wall turbulence. J. Fluid Mech. 225, 213-240.

Jiménez, J., Uhlmann, M., Pinelli, A. \& Kawahara, G. 2001 Turbulent shear flow over active and passive porous surfaces. J. Fluid Mech. 442, 89-117.

Kim, D. \& Chог, H. 2000 A second-order time-accurate finite volume method for unsteady incompressible flow on hybrid unstructured grids. J. Comp. Phys. 162 (2), 411-428.

Kramer, M. 1937 Patentschrift: Einrichtung zur Verminderung des Reibungswiderstandes. Reichspatentamt 1939, Klasse 62b, Gruppe $408 \mathbf{6 6 9 8 9 7 .}$

Letcher, J., Marshall, J., III, J. Oliver \& Salvesen, N. 1987 Stars \& stripes. Scientific American 257 (2).

Linde, P. \& Hegenbart, M. 2019 Riblet film for reducing the air resistance of aircraft. US Patent (US 2019 / 0047684 A1).

Liu, C., Kline, S. \& Johnston, J. 1966 An experimental study of turbulent boundary layer on rough walls. Report, Thermosciences Division, Department of Mechanical Engineering, Stanford University (MD-15).

Liu, K., Christodoulout, C., Ricciust, O. \& Joseph, D. 1990 Drag reduction in pipes lined with riblets. AIAA J. 28 (10), 1697-1698.

Luchini, P. 1996 Reducing the turbulent skin friction. Comp. Methods in App. Sc. 3, 466-470.

Luchini, P., Manzo, F. \& Pozzi, A. 1991 Resistance of a grooved surface to parallel flow and cross-flow. J. Fluid Mech. 228, 87-109.

MacDonald, M., Chan, L., Chung, D., Hutchins, N. \& Ooi, A. 2016 Turbulent flow over transitionally rough surfaces with varying roughness densities. J. Fluid Mech. 804, 130161.

MacDonald, M., Chung, D., Hutchins, N., Chan, L., Ooi, A. \& García-Mayoral, R. 2017 The minimal-span channel for rough-wall turbulent flows. J. Fluid Mech. 816, 5-42.

Martell, M. B., Perot, J. B. \& Rothstein, J. P. 2009 Direct numerical simulations of turbulent flows over superhydrophobic surfaces. J. Fluid Mech. 620, 31-41.

Moin, P. \& Kim, J. 1982 Numerical investigation of turbulent channel flow. J. Fluid Mech. 118, 341-377.

Monty, J. P., Hutchins, N., NG, H. C. H., Marusic, I. \& Chong, M. S. 2009 A comparison of turbulent pipe, channel and boundary layer flows. J. Fluid Mech. 632, 431-442.

Moser, R., Kim, J. \& Mansour, N. 1999 Direct numerical simulation of turbulent channel flow up to $R e_{\tau}=590$. Phys. Fluids 11 (4), 943-945.

Nepf, H. M., Ghisalberti, M., White, B. \& Murphy, E. 2007 Retention time and dispersion associated with submerged aquatic canopies. Water Resour. Res. 43 (4).

Peet, Y., Sagaut, P. \& Charron, Y. 2009 Pressure loss reduction in hydrogen pipelines by surface restructuring. Int. J. Hydrogen Energy 34 (21), 8964-8973.

Poggi, D., Porporato, A., Ridolfi, L., Albertson, J. D. \& Katul, G. G. 2004 The effect of vegetation density on canopy sub-layer turbulence. Boundary-Layer Met. 111 (3), 565587.

Rastegari, A. \& Akhavan, R. 2018 The common mechanism of turbulent skin-friction drag 
reduction with superhydrophobic longitudinal microgrooves and riblets. J. Fluid Mech. 838, 68-104.

Raupach, M. R., Finnigan, J. J. \& Brunet, Y. 1996 Coherent eddies and turbulence in vegetation canopies: The mixing-layer analogy. Boundary-Layer Met. 25, 351-382.

RAYLEIGH, LORD 1879 On the stability, or instability, of certain fluid motions. Proceedings of the London Mathematical Society s1-11 (1), 57-72.

Schrauf, G. 2005 Status and perspectives of laminar flow. Aero. J. 109 (1102), 639-644.

Sharma, A. \& García-Mayoral, R. $2020 a$ Scaling and dynamics of turbulence over sparse canopies. J. Fluid Mech. 888, A1.

Sharma, A. \& García-Mayoral, R. $2020 b$ Turbulent flows over dense filament canopies. J. Fluid Mech. 888, A2.

Singh, R., Bandi, M. M., Mahadevan, A. \& Mandre, S. 2016 Linear stability analysis for monami in a submerged seagrass bed. J. Fluid Mech. 786, R1.

Spalart, P. R. \& McLean, D. 2011 Drag reduction: enticing turbulence, and then an industry. Phil. Trans. R. Soc. A 369, 1556-1569.

Szodruch, J. 1991 Viscous drag reduction on transport aircraft. 29th Aerospace Sciences Meeting (AIAA Paper 1991-685).

Vinuesa, R., Prus, C., Schlatter, P. \& Nagib, H, M. 2016 Convergence of numerical simulations of turbulent wall-bounded flows and mean cross-flow structure of rectangular ducts. Meccanica 51 (12), 3025-3042.

WALsh, M. 1982 Turbulent boundary layer drag reduction using riblets. 20th Aerospace Sciences Meeting (AIAA Paper 1982-169).

Walsh, M. \& Lindemann, A. 1984 Optimization and application of riblets for turbulent drag reduction. 22nd Aerospace Sciences Meeting (AIAA Paper 1984-347).

Walsh, M. \& Weinstein, L. 1978 Drag and heat transfer on surfaces with small longitudinal fins. 11th Fluid and Plasma Dynamics Conference (AIAA Paper 1987-1161).

White, B. L. \& Nepf, H. M. 2007 Shear instability and coherent structures in shallow flow adjacent to a porous layer. J. Fluid Mech. 593, 1-32. 


\section{University Library}

\section{- $\mathrm{M}$ IIN E R VA A gateway to Melbourne's research publications}

Minerva Access is the Institutional Repository of The University of Melbourne

Author/s:

Endrikat, S;Modesti, D;Garcia-Mayoral, R;Hutchins, N;Chung, D

Title:

Influence of riblet shapes on the occurrence of Kelvin-Helmholtz rollers

Date:

2021-03-02

Citation:

Endrikat, S., Modesti, D., Garcia-Mayoral, R., Hutchins, N. \& Chung, D. (2021). Influence of riblet shapes on the occurrence of Kelvin-Helmholtz rollers. JOURNAL OF FLUID MECHANICS, 913, https://doi.org/10.1017/jfm.2021.2.

Persistent Link:

http://hdl.handle.net/11343/267819 